\title{
Ultrafast destruction and recovery of the spin density wave order in iron-based pnictides: A multipulse optical study
}

\author{
M. Naseska, ${ }^{1}$ A. Pogrebna, ${ }^{1,2}$ G. Cao,${ }^{3}$ Z. A. Xu, ${ }^{3}$ D. Mihailovic, ${ }^{1,4}$ and T. Mertelj ${ }^{1,4,}{ }^{*}$ \\ ${ }^{1}$ Complex Matter Department, Jozef Stefan Institute, Jamova 39, Ljubljana, SI-1000 Ljubljana, Slovenia \\ ${ }^{2}$ Institute for Molecules and Materials, Radboud University, Nijmegen 6525 AJ, The Netherlands \\ ${ }^{3}$ Department of Physics, Zhejiang University, Hangzhou 310027, People's Republic of China \\ ${ }^{4}$ CENN Nanocenter, Jamova 39, Ljubljana SI-1000, Slovenia
}

(Received 16 April 2018; revised manuscript received 13 July 2018; published 30 July 2018)

\begin{abstract}
We report on a systematic excitation-density-dependent all-optical femtosecond time-resolved study of the spin density wave state in iron-based superconductors. The destruction and recovery dynamics are measured by means of the standard and a multipulse pump-probe technique. The experimental data are analyzed and interpreted in the framework of an extended three-temperature model. The analysis suggests that the optical-phonon energy relaxation plays an important role in the recovery of almost exclusively electronically driven spin density wave order.
\end{abstract}

DOI: 10.1103/PhysRevB.98.035148

\section{INTRODUCTION}

The collectively ordered electronic states are interesting subjects for driving out of equilibrium by femtosecond optical pulses in order to get better insight into their nature [1-10] and possibly reveal new metastable states [11-13] that are not easily reachable by the quasiequilibrium route. Among such states is also the orthorhombic antiferromagnetic spin density wave- (SDW-) like state in the parent iron-based superconductor compounds [14-16], which is interesting not only due to the proximity to the superconducting state, but also due to its collective itinerant nature and relation to the nematic [17-19] instability.

The ultrafast dynamics of the SDW state in pnictides has been extensively studied by various time-resolved (TR) techniques [20-27]. All-optical [24] and TR angle-resolved photoemission spectroscopy (ARPES) [22] studies show subpicosecond (sub-ps) dynamics with slight slowing down near the transition temperature, whereas the orthorhombic lattice splitting responds much slower [26,27] upon the ultrafast perturbation. An interesting question is what sets the subpicosecond timescale of the suppression and recovery of the electronic SDW order? At weak suppression it appears that the timescale is set by the bottleneck in the relaxation of the nonequilibrium electron distribution function (NEDF) due to the charge gap associated with the SDW order [21,24]. At strong suppression the charge-gap bottleneck is suppressed an the collective SDW dynamics and/or the electron-phonon coupling might play a role in setting the timescale.

In order to improve understanding of the suppression and recovery timescales at strong suppression we conducted a systematic fluence-dependent femtosecond time-resolved alloptical study of the SDW state in two iron-based superconductor parent compounds: $A \mathrm{Fe}_{2} \mathrm{As}_{2}(A=\mathrm{Eu}, \mathrm{Sr})$. In the study we supplemented the standard pump- $(\mathrm{P}-)$ probe $(\mathrm{Pr})$ technique

*tomaz.mertelj@ijs.si with the multipulse technique that proved to be instrumental $[9,28]$ to obtain insight into the collective dynamics in chargedensity wave systems [28] and superconductors [9].

To identify the processes that set the SDW recovery time we analyze the multipulse data in the framework of an extended three-temperature model (3TM). Surprisingly, the 3TM analysis suggests that an excitation-density-dependent optical-phonons (OPs)-lattice-bath energy-relaxation bottleneck plays a crucial role in the NEDF relaxation and the SDW order recovery, whereas the collective SDW order dynamics is too fast to influence the dynamics beyond $\sim 200 \mathrm{fs}$. Moreover, the resilience of the SDW state to strong ultrafast optical excitation is suggested to be a consequence of a fast electron-opticalphonon energy transfer during the initial NEDF thermalization on a few hundred femtosecond timescale that is enhanced at high-excitation densities.

\section{EXPERIMENT}

\section{A. Samples}

Single crystals of $\mathrm{EuFe}_{2} \mathrm{As}_{2}$ (Eu-122) and $\mathrm{SrFe}_{2} \mathrm{As}_{2}$ (Sr122) were grown at Zhejiang University by a flux method as described previously [24]. In both compounds the onset of the antiferromagnetic SDW-like ordering is concurrent with the structural transition from tetragonal to orthorhombic symmetry $T_{\mathrm{N}}=190 \mathrm{~K}$ for Eu-122 [29] and $T_{\mathrm{N}}=203 \mathrm{~K}$ for Sr-122 [29].

\section{B. Optical setup}

Measurements of the multipulse transient reflectivity were performed using an extension of the standard pump-probe technique with $\sim 50$-fs optical pulses from either 1- or 250 $\mathrm{kHz}$ Ti: $\mathrm{Al}_{2} \mathrm{O}_{3}$ regenerative amplifiers seeded with Ti: $\mathrm{Al}_{2} \mathrm{O}_{3}$ oscillators. The output-pulse train was split into destruction(D-), P-, and Pr-pulse trains that were independently delayed with respect to each other. The P- and D-pulse beams were either at the laser fundamental $\left(\hbar \omega_{\mathrm{P}}=1.55-\mathrm{eV}\right)$ or the doubled 


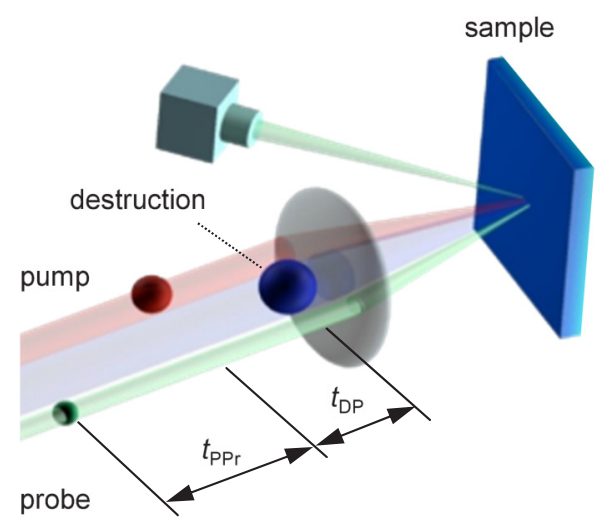

FIG. 1. Schematic of the three-pulse experiment and notation of the delays between pulses.

$\left(\hbar \omega_{\mathrm{P}}=3.1-\mathrm{eV}\right)$ photon energy, whereas the Pr beam was always at the laser fundamental $\hbar \omega_{\mathrm{pr}}=1.55-\mathrm{eV}$ photon energy.

The resulting beams were focused and overlapped on the sample (see Fig. 1). As in the standard pump-probe stroboscopic experiments the multipulse transient reflectivity $\Delta R_{3} / R$ was measured by monitoring the intensity of the weakest $\mathrm{Pr}$ beam. The direct contribution of the unchopped $\mathrm{D}$ beam to the total transient reflectivity $\Delta R$ was rejected by means of a lock in amplifier synchronized to the chopper that modulated the intensity of the P beam only. The fluences of the P- and Pr-pulses $\mathcal{F}_{\mathrm{Pr}}<\mathcal{F}_{\mathrm{P}} \lesssim 100 \mu \mathrm{J} / \mathrm{cm}^{2}$ were kept in the linearresponse region.

Due to the chopping scheme the measured quantity in the multipulse experiments is the difference between the transient reflectivity in the presence of $\mathrm{P}$ - and D-pulses $\Delta R_{\mathrm{DP}}\left(t_{\mathrm{Pr}}, t_{\mathrm{P}}, t_{\mathrm{D}}\right)$ and the transient reflectivity in the presence of the $\mathrm{D}$ pulse only $\Delta R_{\mathrm{D}}\left(t_{\mathrm{Pr}}, t_{\mathrm{D}}\right)$,

$$
\Delta R_{3}\left(t_{\mathrm{Pr}}, t_{\mathrm{P}}, t_{\mathrm{D}}\right)=\Delta R_{\mathrm{DP}}\left(t_{\mathrm{Pr}}, t_{\mathrm{P}}, t_{\mathrm{D}}\right)-\Delta R_{\mathrm{D}}\left(t_{\mathrm{Pr}}, t_{\mathrm{D}}\right),
$$

where $t_{\mathrm{Pr}}, t_{\mathrm{P}}$, and $t_{\mathrm{D}}$ correspond to the Pr-, P-, and D-pulse arrival times, respectively.
When using the doubled P-photon energy the scattered pump photons were rejected by long-pass filtering, whereas an analyzer oriented perpendicularly to the P-beam polarization was used for rejection in the case of the degenerate P- and Pr-photon energies. All beams were nearly perpendicular to the cleaved sample surface (001). Both the $\mathrm{P}$ and the $\mathrm{D}$ beams had polarizations perpendicular to the polarization of the $\mathrm{Pr}$ beam, which was oriented with respect to the crystals to obtain the maximum or minimum amplitude of the sub-picosecond $\Delta R / R$ at low temperatures. The pump beam diameters were, depending on experimental conditions, in a (50-100)- $\mu \mathrm{m}$ range with somewhat smaller probe beam diameters. The beam diameters were determined either by a direct measurement of the profile at the sample position by means of a complementary metal-oxide semiconductor camera or by measuring the transmission through a set of calibrated pinholes.

\section{STANDARD PUMP-PROBE RESULTS}

As noted previously [30] we observe a twofold rotational anisotropy of the transient reflectivity with respect to the probe polarization with different orientations in different domains. To measure a single domain dominated response the positions on the sample surface with maximal anisotropy of the response have been chosen for measurements. In the absence of information about the in-plane crystal axes orientation in the chosen domains we denote the probe-polarization orientation according to the polarity of the observed sub-picosecond low $T$ response as $\mathcal{P}^{+}$and $\mathcal{P}^{-}$. The magnitude of the $\mathcal{P}^{-}$ response is larger than the magnitude of the $\mathcal{P}^{+}$response in both compounds, so in the multipulse experiments the $\mathcal{P}^{-} \mathrm{Pr}$ polarization was used in most of the cases.

\section{A. Fluence dependence}

In Fig. 2 we plot the fluence dependence of the standard two-pulse transient reflectivity in the SDW state. In both compounds we observe a linear scaling of $\Delta R / R$ with the pump fluence $\left(\mathcal{F}_{\mathrm{P}}\right)$ up to the threshold fluence $\mathcal{F}_{\mathrm{th}} \sim 0.2 \mathrm{~mJ} / \mathrm{cm}^{2}$.
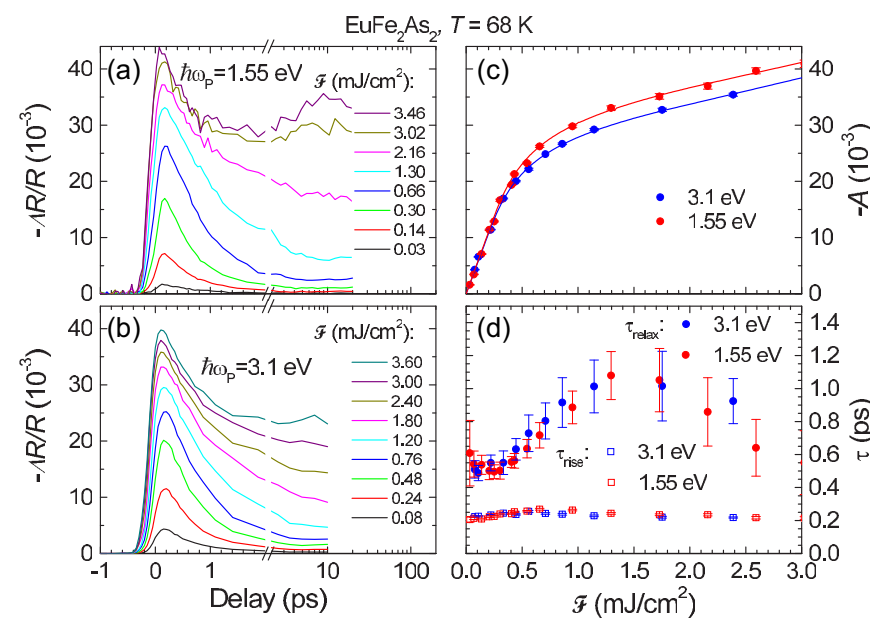

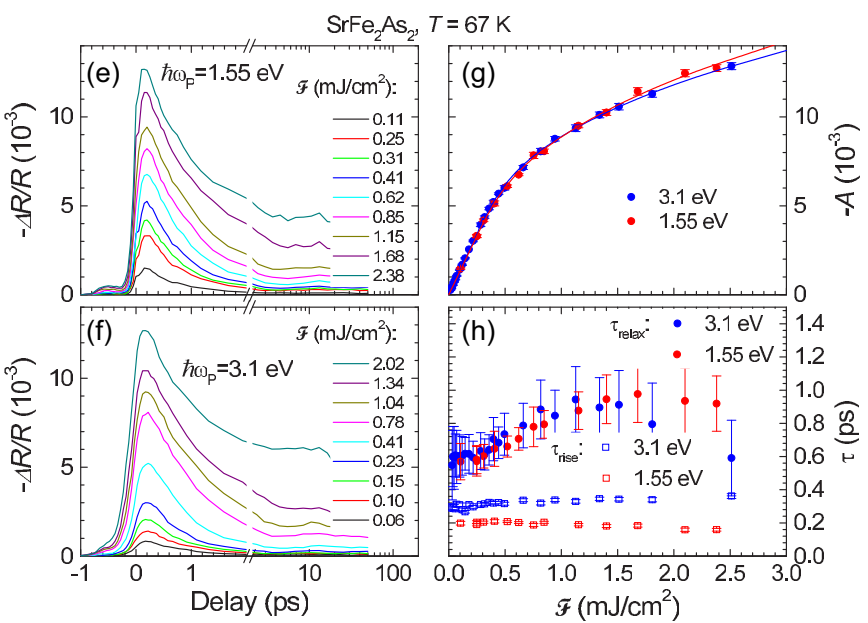

FIG. 2. Fluence dependence of the transient reflectivity in the SDW state for the $\mathcal{P}^{-}$probe polarization in (a) and (b) Eu-122 and (e) and (f) Sr-122 at two different pump-photon energies. The amplitude of the transient reflectivity as a function of the pump fluence in (c) Eu-122 and (g) Sr-122. The corresponding transient reflectivity decay and rise times as a function of the pump fluence are shown in (d) and (h). The thin lines in (c) and (g) are the saturation model fits discussed in the text. 


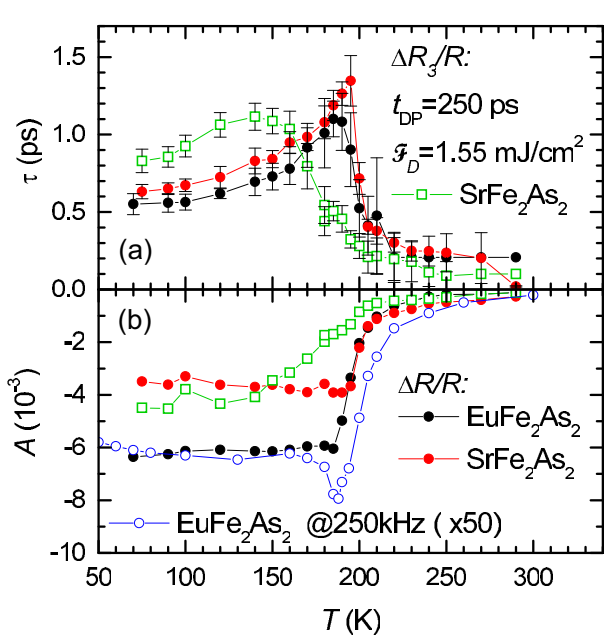

FIG. 3. Temperature dependence of the relaxation time and amplitude for the $\mathcal{P}^{-}$polarization measured at a $1-\mathrm{kHz}$ pulse repetition rate in the absence (red and black full circles) and presence of the D-pulse heating (green open squares). For comparison we plot also the amplitude $T$ dependence measured at a $250-\mathrm{kHz}$ repetition rate (blue open circles) at $\sim 50$ times lower pump fluence.

Above this value the amplitude of the initial sub-ps transient shows a partial saturation increasing linearly with a different slope and nonzero intercept above $\mathcal{F}_{\mathrm{P}} \sim 1 \mathrm{~mJ} / \mathrm{cm}^{2}$. In this region of fluence also a long-lived component following the initial sub-ps transient becomes rather prominent.

The rise time of the transients $\tau_{\text {rise }}$ shows no fluence dependence whereas the initial sub-ps decay time rises from the below- $\mathcal{F}_{\text {th }}$ value of $\tau_{\text {relax }} \sim 0.6$ ps to a maximum value of $\tau_{\text {relax }} \sim 1$ ps at $\mathcal{F}_{\mathrm{P}} \sim 1.5 \mathrm{~mJ} / \mathrm{cm}^{2}$ decreasing back to $\tau_{\text {relax }} \sim$ $0.6 \mathrm{ps}$ at the highest $\mathcal{F}_{\mathrm{P}} \sim 3 \mathrm{~mJ} / \mathrm{cm}^{2}$.

\section{B. Transient heating}

In order to experimentally assess the transient thermal heating of the experimental volume we measured the temperature dependence of $\Delta R_{3} / R$ in Sr-122 at $\mathcal{F}_{\mathrm{D}}=1.55 \mathrm{~mJ} / \mathrm{cm}^{2}$ and long $t_{\mathrm{DP}} \sim 250 \mathrm{ps}$ [31] and compared it to temperature dependence of $\Delta R / R$ in the absence of the $D$ pulse. From Fig. 3 we can see that in the absence of the D pulse the relaxation time shows a characteristic [24] $T$ dependence and can be used as a proxy to the temperature to estimate the transient lattice heating in the presence of the D pulse. In the presence of the D pulse the characteristic relaxation-time peak at $T_{\mathrm{N}}$ is shifted $\sim 60 \mathrm{~K}$ towards lower temperatures and smeared due to the temperature gradient perpendicular to the sample surface. The experimental thermal heating at $T \sim 150 \mathrm{~K}$ is therefore $\Delta T \sim 60 \mathrm{~K}$ at $\mathcal{F}_{\mathrm{D}}=1.55 \mathrm{~mJ} / \mathrm{cm}^{2}$ increasing to $\Delta T \sim 90 \mathrm{~K}$ at $T \sim 70 \mathrm{~K}$.

On the other hand, taking into account the experimental temperature-dependent specific heat capacity $[32,33]$ and optical [34,35] data we estimate [36] (at $T \sim 70 \mathrm{~K}$ ) a temperature increase of $\Delta T \sim 210 \mathrm{~K}$ at the fluence $\mathcal{F}_{\mathrm{D}}=1.55 \mathrm{~mJ} / \mathrm{cm}^{2}$, whereas the transition temperature of $T_{\mathrm{N}} \sim 200 \mathrm{~K}$ would be reached at $\mathcal{F}_{\mathrm{P}} \sim 1 \mathrm{~mJ} / \mathrm{cm}^{2}$. The estimated $\Delta T$ is therefore more than two times larger than the directly measured.

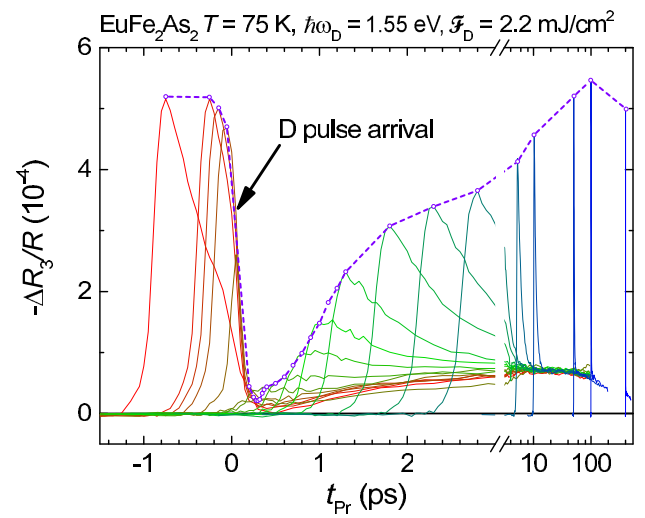

FIG. 4. The $\mathcal{P}^{-}$multipulse transient reflectivity in Eu-122 in the presence of the destruction pulse arriving at $t_{\mathrm{D}}=0 \mathrm{ps}$ for different pump-pulse arrival times measured with the $250-\mathrm{kHz}$ repetition rate laser system. The dashed line represents the trajectory defined in the text.

\section{MULTIPULSE RESULTS}

\section{A. Multipulse trajectories}

In Fig. 4 we plot results of a typical multipulse experiment where the destruction pulse arrives at $t_{\mathrm{D}}=0 \mathrm{ps}$, whereas the pump-pulse and probe-pulse arrival times are varied. By tracking the value of $\Delta R_{3}\left(t_{\mathrm{Pr}}, t_{\mathrm{P}}, t_{\mathrm{D}}\right)$ at a constant $t_{\mathrm{PPr}}=t_{\mathrm{Pr}}-t_{\mathrm{P}}$ at the extremum $\left(t_{\mathrm{PPr}} \sim 200 \mathrm{fs}\right)$ of the unperturbed [37] $\Delta R / R$ (Fig. 5), we define the trajectory $A_{3}\left(t_{\mathrm{DP}}\right)$, where $t_{\mathrm{DP}}=t_{\mathrm{P}}-t_{\mathrm{D}}$ is the delay between the $\mathrm{D}$ and the $\mathrm{P}$ pulses. Due to the finite $t_{\mathrm{PPr}}$ at the readout of $A_{3}\left(t_{\mathrm{DP}}\right)$ the temporal resolution of the trajectory is limited to $\sim t_{\mathrm{PPr}} \approx 200 \mathrm{fs}$.

In Figs. 6(a) and 6(c) we plot typical trajectories for both probe polarizations at $T \sim 70 \mathrm{~K}$. Below $\mathcal{F}_{\mathrm{D}} \sim 1 \mathrm{~mJ} / \mathrm{cm}^{2}$ the trajectories indicate a recovery of the ordered state on the subps timescale. Above $\mathcal{F}_{\mathrm{D}} \sim 2 \mathrm{~mJ} / \mathrm{cm}^{2}$ the recovery timescale slows down beyond hundreds of picoseconds. In the intermediate region $1 \mathrm{~mJ} / \mathrm{cm}^{2} \lesssim \mathcal{F}_{\mathrm{D}} \lesssim 2 \mathrm{~mJ} / \mathrm{cm}^{2}$ the recovery is still observed on a few ps timescale. Since the heat cannot diffuse out of the excited sample volume on this timescale this

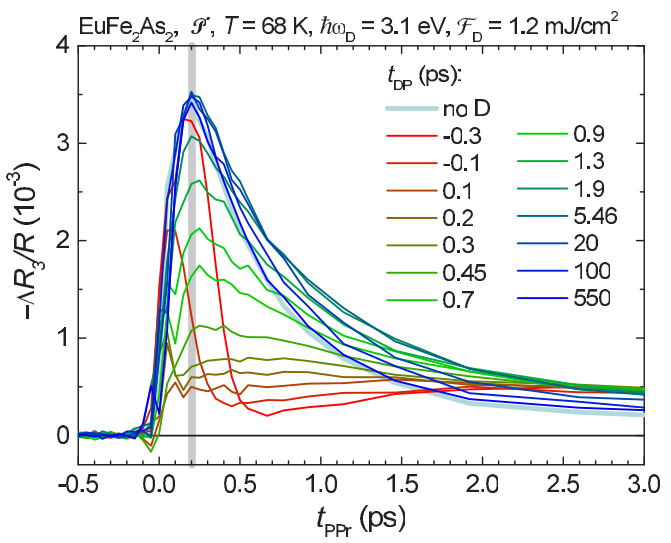

FIG. 5. Example of the multipulse transient reflectivity in Eu-122 at different $t_{\mathrm{DP}}$ relative to the pump arrival time $t_{\mathrm{P}}$ measured with the $1-\mathrm{kHz}$ repetition rate laser system. The vertical gray line indicates the trajectory readout pump-probe delay. 

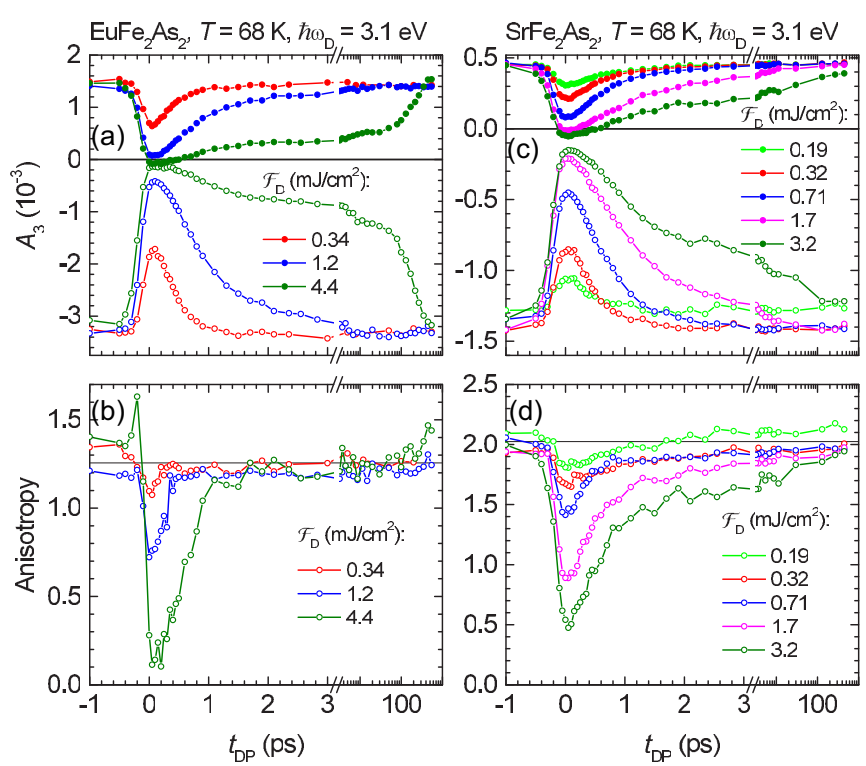

FIG. 6. The trajectories for the $\mathcal{P}^{+}$(full symbols) and $\mathcal{P}^{-}$(open symbols) polarizations at a few characteristic destruction-pulse fluences in (a) Eu-122 and (c) Sr-122. Anisotropy (see the text for a definition) of the trajectories at different destruction fluences in (b) Eu-122 and (d) Sr-122.

indicates that the transient lattice temperature does not exceed $T_{\mathrm{N}}$ below $\mathcal{F}_{\mathrm{D}} \sim 2 \mathrm{~mJ} / \mathrm{cm}^{2}$. This fluence therefore represents the boundary between the fast-quench and the slow-quench conditions.

In Figs. 6(b) and 6(d) we plot also the anisotropy defined as $\left(A_{3 \mathcal{P}_{+}}-A_{3 \mathcal{P}_{-}}\right) /\left(A_{3 \mathcal{P}_{+}}+A_{3 \mathcal{P}_{-}}\right)$. In Eu-122 the anisotropy recovers on the sub-ps timescale even at the slow-quench conditions whereas in Sr-122 the initial sub-ps recovery is followed by a slower tail lasting more than $\sim 10 \mathrm{ps}$.

In Fig. 7 we also compare the trajectories to the standard transient reflectivity measured at similar excitation fluences. In the case of fast-quench $\mathcal{F}_{\mathrm{D}} \lesssim 2 \mathrm{~mJ} / \mathrm{cm}^{2}$, the trajectories recover faster than the corresponding transient reflectivity for both D-photon energies. In the case of extremely slow-quench $\mathcal{F}_{\mathrm{D}} \gtrsim 2 \mathrm{~mJ} / \mathrm{cm}^{2}$, the trajectory dynamics shows only the slow
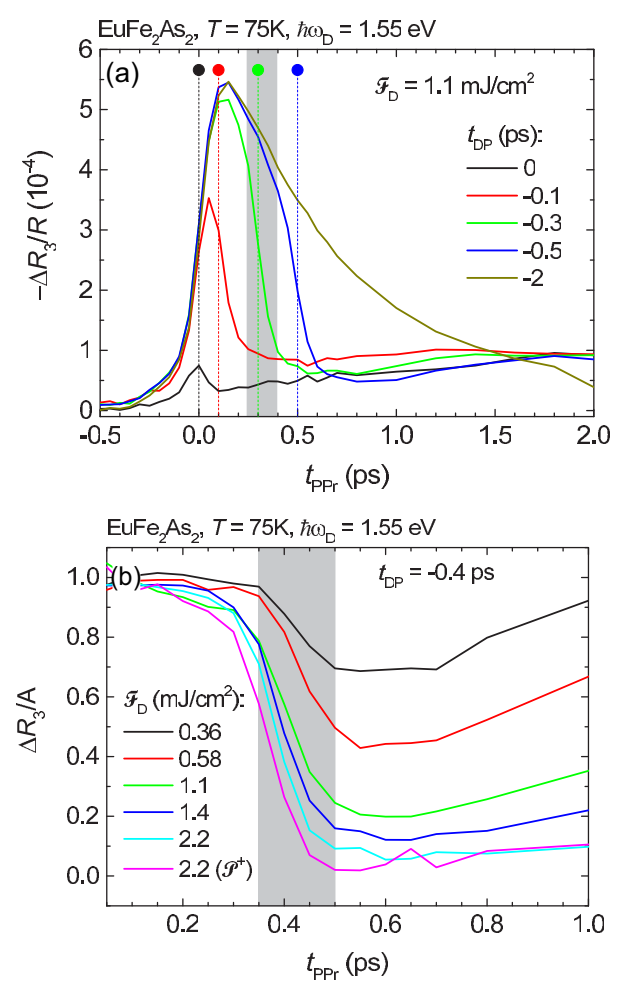

FIG. 8. (a) The $\mathcal{P}^{-}$multipulse transient reflectivity in Eu-122 at different destruction-pulse arrival times $t_{\mathrm{DP}}$. The timescale of the transient reflectivity suppression region of $150 \mathrm{fs}$ for $t_{\mathrm{DP}}=0.3 \mathrm{ps}$ is indicated by the shaded region. (b) The relative suppression of $\mathcal{P}^{-} \Delta R_{3}$ as a function of $\mathcal{F}_{\mathrm{D}}$ for the destruction pulse arriving at $t_{\mathrm{DP}}=-0.4 \mathrm{ps}$.

recovery whereas the transient reflectivity still displays a partial initial sub-picosecond relaxation.

\section{B. Destruction timescale}

To determine the destruction timescale of the ordered state we chose a negative $t_{\mathrm{DP}}$ and analyze the suppression of $\Delta R_{3} / R$ after the D-pulse arrival. As shown in Fig. $8 \Delta R_{3} / R$ is suppressed within $\sim 150$ fs at $1.55-\mathrm{eV}$ D-photon energy. The
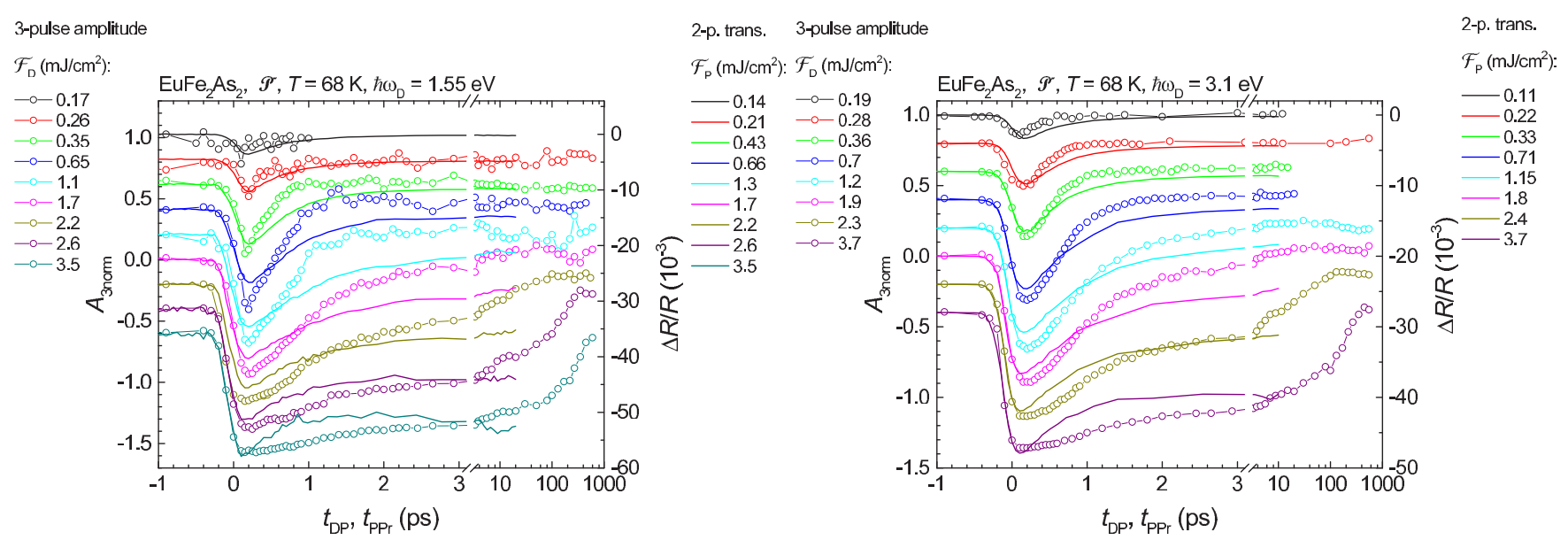

FIG. 7. Comparison of the normalized $\mathcal{P}^{-}$trajectories (open symbols) to the $\mathcal{P}^{-}$transient reflectivity (full lines) at different destruction/pump fluences in Eu-122 for two different pump/destruction photon energies. 

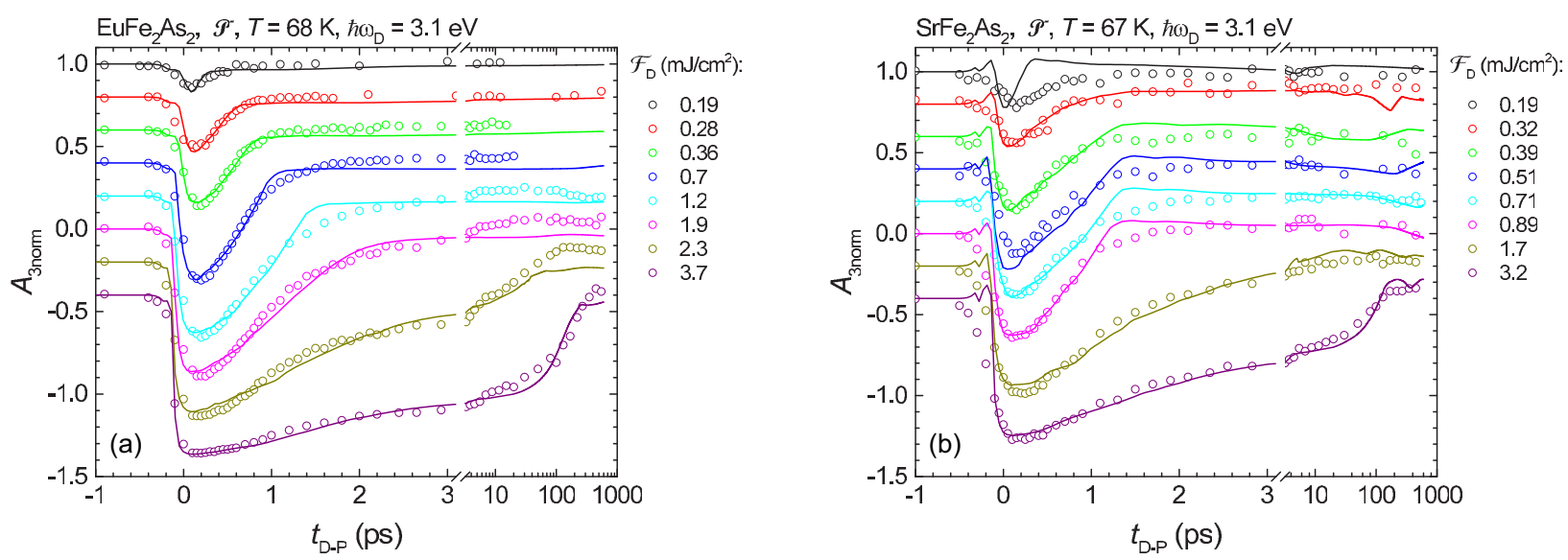

FIG. 9. Comparison of the experimental (open circles) and simulated (lines) trajectories for the case of $\phi=\pi / 2$. The trajectories at different $\mathcal{F}_{\mathrm{D}}$ 's are vertically shifted for clarity.

suppression timescale does not depend on $\mathcal{F}_{\mathrm{D}}$, although we observe an earlier onset of the suppression at higher $\mathcal{F}_{\mathrm{D}}$. The effect can be attributed to the wing of the $\mathrm{D}$ pulse extending beyond $\sim 50$ fs that at higher $\mathcal{F}_{\mathrm{D}}$ contains enough energy to start the ordered-state suppression prior to the arrival of the central part of the D pulse.

Comparing the trajectories measured at different destruction-photon energies in Fig. 7 we observe a sharper feature around the maximal-suppression $t_{\mathrm{DP}}$ in the case of the degenerate $\hbar \omega_{\mathrm{D}}=1.55-\mathrm{eV}$ D-photon energy. Whereas the suppression timescale appears identical for both D-photon energies in Eu-122 the suppression at 3.1-eV D-photon energy in Sr-122 appears slower [Fig. 9(b)] consistent with the slower rise time in the standard pump-probe experiment [Fig. 2(h)].

\section{ANALYSIS AND DISCUSSION}

\section{A. Destruction-pulse absorption saturation}

The large difference between experimentally determined transient lattice heating and the estimate based on the equilibrium optical and thermodynamic properties indicates that the D-pulse energy of $\sim 250 \mathrm{ps}$ after the pulse arrival is deposited in a layer that is $\sim 3$ times thicker than the optical penetration depth $(\sim 20-30 \mathrm{~nm})$ at the highest fluences used. This large energy deposition depth can neither be accounted for by the thermal diffusion [38] on the $\sim 250$-ps timescale nor the initial ballistic photoexcited carrier transport [39,40]. The most plausible explanation that remains is therefore saturation of absorption. This is supported also by the fact that the multipulse trajectories (see Fig. 7) show a sharp feature near $t_{\mathrm{DP}}=0$ when the pump and destruction photon energies are degenerate.

\section{B. Anisotropy recovery}

As discussed previously [41] the observed transientreflectivity anisotropy can be connected to the orbital degrees of freedom, consistent with ARPES [42]. The quick suppression and sub-picosecond recovery of the anisotropy [see Figs. 6(b) and 6(d)] at any fluence additionally confirm that the transient reflectivity is dominated by the electronic response and that the observed optical symmetry breaking is predominantly related to the fluctuating orbital degrees of freedom with a negligible lattice contribution.

\section{Three-temperature model simulations of recovery}

In all-optical experiments it is generally not possible to directly disentangle dynamics of different degrees of freedom due to unknown response functions. In general, both $\Delta R$ and $A_{3}$ can couple to single-particle and order parameter excitations. We therefore seek better insight into the recovery by means of semiempirical simulations similar as previously in the cuprate superconductors $[9,43]$.

At low-excitation densities the order parameter as well as $\Delta R$ can usually be linearly expanded in terms of a single parameter [44] that is used to describe the NEDF dynamics. In the present compounds we have conjectured that the low-excitation transient reflectivity couples to the collective SDW order parameter that has fast femtosecond-timescale dynamics. In such a case the order parameter and transient reflectivity directly follow the magnon-bottleneck governed NEDF dynamics [24].

At high-excitation densities the relation between the NEDF and the order parameter becomes nonlinear, and the simple low-excitation description of $\Delta R$ is expected to break down. This is indicated by the difference between the relaxation dynamics (Fig. 7) observed in the standard pump-probe and multipulse experiments that suggests that NEDF and the order parameter have different delay dependences.

In the cuprate superconductors the characteristic timescale of the order parameter relaxation appears to be $[9,43]$ on a picosecond timescale, and the intrinsic order parameter dynamics plays an important role on the experimental-observation timescale. In the present case the SDW order parameter is expected to relax much faster due to a larger gap $\left(2 \Delta_{\text {SDW }} \sim\right.$ $200 \mathrm{meV}$ [24]). An estimate of the SDW amplitude mode frequency [45] $\omega_{\mathrm{AM}}=2 \Delta_{\mathrm{SDW}} / \hbar$ would lead to the relaxation timescale bottom limit of $\sim 3$ fs. On the other hand, the SDW transition is coupled to the structural transition that could lead to renormalization and slowdown of the order parameterrelaxation timescale. Recent time-resolved x-ray-diffraction experiments [26,27] showed, however, that on the tens of 
picoseconds timescale the orthorhombic lattice splitting is decoupled from the electronic order parameter.

In the present multipulse experiments the time resolution of the trajectories is not better than the rise time of the standard pump-probe response ( $\sim 200 \mathrm{fs})$. Any intrinsic order parameter dynamics faster than $\sim 200$ fs would therefore not be revealed in the experiment. Since it is very likely that the intrinsic order parameter-relaxation timescale is faster than the resolution we check this hypothesis by simulating the trajectories assuming that the order parameter and the optical response directly follow the NEDF on the experimentally accessible timescales.

Since modeling of the NEDF dynamics in strongly excited collectively ordered systems, such as SDWs, is prohibitively difficult we further assume [46] that NEDF can be approximately described by an electronic temperature. To calculate the optical response we use an empirical response function assuming and that the amplitude of the pump-pulse-induced transient dielectric constant $\Delta \epsilon_{3}^{\mathrm{A}}\left(z, t_{\mathrm{DP}}\right)$ depends on the local electronic temperature $T_{\mathrm{e}}\left(z, t_{\mathrm{DP}}\right)$ only,

$$
\Delta \epsilon_{3}^{\mathrm{A}}\left(z, t_{\mathrm{DP}}\right) \propto A\left(T_{\mathrm{e}}\left[z, t_{\mathrm{DP}}\right]\right) .
$$

Here $A(T)$ is the experimental $T$-dependent amplitude measured in the absence of the D pulse shown in Fig. 3(b), and $z$ corresponds to the normal distance from the sample surface. For the sake of simplification any radial dependence is neglected.

In the present experiment $A(T)$ is measured under near equilibrium conditions where the lattice and electronic components of the order parameter are changing simultaneously with $T$ whereas on the multipulse experiment timescales the lattice and electronic part become decoupled. This can break the validity of assumption (2) if the direct lattice contribution to $A(T)$ is significant. However, indirect evidence suggests that the direct lattice contribution is relatively small. The weak-excitation transient reflectivity shows a sub-picosecond recovery with rather fast 150 -fs rise time and no coherent oscillations that are usually observed when the lattice contribution is significant. Moreover, the transient reflectivity can be almost completely suppressed (and recovers) on the timescale much faster than the measured lattice order parameter response timescale [26,27]. The direct lattice contribution to $A(T)$ can therefore be considered small and almost time independent on the most relevant few-picoseconds timescale.

The multipulse transient reflectivity amplitude is given by (see the Appendix)

$$
A_{3}\left(t_{\mathrm{DP}}\right) \propto \int_{0}^{\infty} d z e^{-\alpha_{\mathrm{Pr}} z} \cos \left(2 n_{\mathrm{Pr}} \frac{\omega_{0}}{c_{0}} z-\phi\right) \Delta \epsilon_{3}^{\mathrm{A}}\left(z, t_{\mathrm{DP}}\right),
$$

where $\alpha_{\mathrm{Pr}}$ and $n_{\mathrm{Pr}}$ are the probe absorption coefficient and the real part of the refraction index, respectively. The phase $\phi$ depends [see Appendix Eq. (A11)] on the static complex refraction index and the ratio between the real and the imaginary parts of $\Delta \epsilon_{3}^{\mathrm{A}}$.

Since $A(T)$ is virtually temperature independent in the SDW state dropping abruptly above $T_{\mathrm{SDW}}$, the trajectories $A_{3}\left(t_{\mathrm{DP}}\right)$ are expected to reflect mainly the SDW volumefraction dynamics in the probed volume [47] and/or the normal-/nematic-state dynamics when the SDW state is completely suppressed. Since $A(T) \rightarrow 0$ above $\sim 300 \mathrm{~K}$ the sensitivity in the later case is limited to the temperature window between $\sim 200$ and $\sim 300 \mathrm{~K}$.

The evolution of $T_{\mathrm{e}}\left(z, t_{\mathrm{DP}}\right)$ after the $\mathrm{D}$ pulse is calculated solving a three-temperature model $[46,48]$ where a subset of optical phonons with temperature $T_{0}$, different from the lattice temperature $T_{\mathrm{L}}$, is assumed to be strongly coupled to the electronic subsystem [49]. In the simplest 3TM it is also assumed that all the absorbed energy remains in the electronic subsystem until it completely thermalizes. Here the model is extended assuming that a part $\eta$ of the absorbed energy is transferred directly to a few strongly coupled optical phonons during thermalization of the NEDF on a few-100-fs timescale [50].

To fit the simulated trajectories to the experimental data we take the experimental specific-heat-capacity $c_{\mathrm{p}}(T)[32,33]$ and set $\phi$ in Eq. (3) to either 0 or $\pi / 2$. We also fix the heating pulse length to $200 \mathrm{fs}$ corresponding to the trajectory temporal resolution. The rest of the 3TM parameters are determined from the nonlinear least-squares fit. In the first step only the trajectories for the highest $\mathcal{F}_{\mathrm{D}}$ are fit. Due to rather accurate total specific-heat-capacity $[32,33]$ and static optical-reflectivity data $[34,35]$, the thermal conductivity $k$ and the phenomenological D-pulse saturated-absorption length $L_{\mathrm{D}} \sim 80 \mathrm{~nm}[51]$ at the highest $\mathcal{F}_{\mathrm{D}}$ are obtained from the long DP-delay behavior in both samples.

To fit the lower- $\mathcal{F}_{\mathrm{D}}$ trajectories it is assumed that $L_{\mathrm{D}} \propto \mathcal{F}_{\mathrm{D}}$ [52] whereas a global fit over all trajectories at different $\mathcal{F}_{\mathrm{D}}$ 's is used to determine the remaining parameters. With all the fit parameters taken to be independent of $\mathcal{F}_{\mathrm{D}}$ it is not possible to obtain reasonable fits at all experimental $\mathcal{F}_{\mathrm{D}}$ 's simultaneously since the 3TM model results in a too strong slowdown of the relaxation with increasing $\mathcal{F}_{\mathrm{D}}$. On the other hand, assuming that the optical-phonon-lattice-relaxation rate $G_{\mathrm{ol}}$ and $\eta$ depend on $\mathcal{F}_{\mathrm{D}}$ and setting $\phi=\pi / 2$ [53] results in excellent fits (shown in Fig. 9) in the complete experimental $\mathcal{F}_{\mathrm{D}}$ range. The quality of the fits supports the initial hypothesis of the fast sub-200-fs order parameter dynamics.

The obtained 3TM fit parameters are shown in Table I and Fig. 10. For comparison the fit parameters from fits to the published TR-ARPES [46] surface- $T_{\mathrm{e}}$ dynamics in Eu-122 are also shown.

The obtained normal-state values of the electronic specificheat constant $\gamma_{\mathrm{e}}$ in the $50-60 \mathrm{~mJ} \mathrm{~mol}^{-1} \mathrm{~K}^{-2}$ range (Table I) are significantly larger than the low-temperature (SDW-state) thermodynamic value of $\gamma_{\mathrm{e}} \sim 8 \mathrm{~mJ} \mathrm{~mol}^{-1} \mathrm{~K}^{-2}[33,55]$. The increase in $\gamma_{\mathrm{e}}$ in the normal state is consistent with the suppression of the SDW gap but appears somewhat larger than upon suppression of the SDW state by Co doping [56] in Ba-122, where $\gamma_{\mathrm{e}}$ increases from $\sim 5 \mathrm{~mJ} \mathrm{~mol}^{-1} \mathrm{~K}^{-2}$ in the SDW state to $\sim 25 \mathrm{~mJ} \mathrm{~mol}^{-1} \mathrm{~K}^{-2}$ in the superconducting samples. On the other hand, assuming that the high normal-state magnetic susceptibility [57] is dominated by the Pauli contribution and the electron-phonon coupling constant is small $[21,41]$ results in comparable $\gamma_{\mathrm{e}} \sim 60 \mathrm{~mJ} \mathrm{~mol}^{-1} \mathrm{~K}^{-2}$.

Although the values of $\gamma_{\mathrm{e}}$ for Eu-122 obtained from our data and TR-ARPES are consistent, there is a much larger discrepancy of the other parameters. Fits to the multipulse 
TABLE I. Comparison of the three-temperature model parameters obtained from fits. $\lambda\left\langle\omega^{2}\right\rangle$ is the second moment of the Eliashberg function proportional [54] [see also Eq. (A16)] to the electron phonon-relaxation rate $G_{\mathrm{eo}}$. The detailed definition of the parameters is given in Appendix Sec. A 2 .

\begin{tabular}{|c|c|c|c|c|c|c|c|c|}
\hline Eu-122 (time-resolved & 0.01 & $52 \pm 3$ & $34 \pm 3$ & $39 \pm 3$ & $67 \pm 14$ & $7 \pm 5$ & - & 17 \\
\hline $\begin{array}{l}\text { ARPES) [46] } \\
(T \sim 100 \mathrm{~K})^{\mathrm{d}}\end{array}$ & 0.5 & $30 \pm 2$ & $23 \pm 3$ & $45 \pm 3$ & $76 \pm 20$ & $11 \pm 9$ & - & 17 \\
\hline $\begin{array}{l}\text { Eu-122 (present } \\
\text { paper })(T \sim 70 \mathrm{~K})\end{array}$ & Fig. 10 & $49 \pm 1$ & $13 \pm 1$ & $16 \pm 2$ & $10 \pm 1$ & Fig. 10 & $14 \pm 1$ & $17 \pm 5$ \\
\hline $\begin{array}{l}\text { Sr-122 (present } \\
\text { paper })(T \sim 70 \mathrm{~K})\end{array}$ & Fig. 10 & $57 \pm 2$ & $16 \pm 1$ & $17 \pm 2$ & $24 \pm 1$ & Fig. 10 & $10 \pm 1$ & $23 \pm 5$ \\
\hline
\end{tabular}

${ }^{\mathrm{a}}$ Fixed at the selected values for the case of TR-ARPES.

${ }^{\mathrm{b}} T_{\mathrm{E}}$ was without fitting set to $300 \mathrm{~K}$.

${ }^{\mathrm{c}}$ Obtained from the two highest fluences fit in the multipulse case.

${ }^{\mathrm{d}} \mathcal{F}_{\mathrm{D}} \sim 1 \mathrm{~mJ} / \mathrm{cm}^{2}$.

trajectories result in a smaller electron-phonon-relaxation rate $G_{\text {eo }}$, larger optical-phonon-lattice-relaxation rate $G_{\text {ol }}$, and significantly smaller strongly coupled optical-phonon heatcapacity $c_{\mathrm{E} 0}$. Partially this can be attributed to the systematic errors of the $3 \mathrm{TM}$ and the response function. Setting $\eta$ to a fixed $\mathcal{F}_{\mathrm{D}}$-independent value results in qualitatively similar trajectories (see the Appendix, Fig. 13 and Table III) with similar $\gamma_{\mathrm{e}}$ 's and $c_{\mathrm{E} 0}$ 's but significantly different relaxation rate parameters. Another obvious contribution to the difference are differences between the surface and the bulk since the present technique is more bulk sensitive than TR-ARPES.

By using a simpler two-temperature model with $\mathcal{F}_{\mathrm{D}^{-}}$ dependent $\gamma_{\mathrm{e}}$ and the electron-phonon coupling it is also possible to obtain fair fits to the trajectories (not shown). However, from such fits an nonphysically large $\gamma_{\mathrm{e}}$ of $\sim 200 \mathrm{~mJ} \mathrm{~mol}^{-1} \mathrm{~K}^{-2}$ is obtained indicating that some strongly coupled optical phonons must play a role in the energy relaxation. It therefore appears that the dominant relaxation bottleneck is cooling of the strongly coupled optical phonons to the lattice bath.

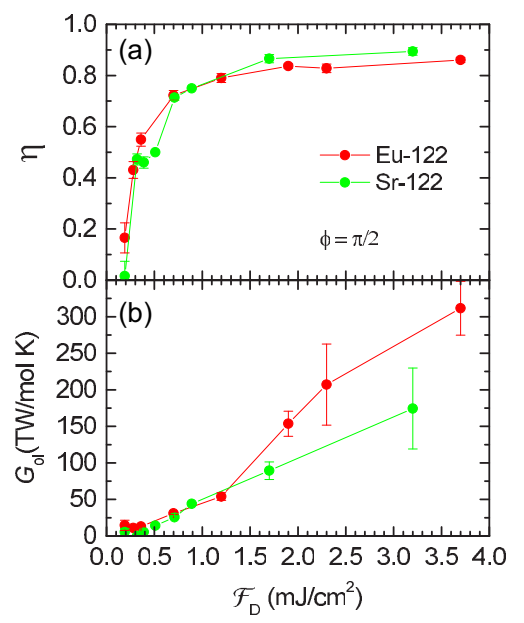

FIG. 10. The destruction-pulse fluence dependence of (a) the branching factor and (b) the electron-optical-phonons coupling from the 3TM fit.
$\mathcal{F}_{\mathrm{D}}$ dependence of the optical-phonons-lattice-relaxation rate $G_{\mathrm{ol}}$ shows a strong increase with increasing $\mathcal{F}_{\mathrm{D}}$ (see Fig. 10). The increase is robust to the variations of the branching-factor fitting approach (see the Appendix, Fig. 14) and can be attributed to opening of additional electronic relaxation channels upon suppression of the nematic-fluctuationsrelated pseudogap [41] in addition to the anharmonic-decay channels.

A less robust [58] result of our analysis is the increase in the branching factor $\eta$ with increasing $\mathcal{F}_{\mathrm{D}}$ suggesting that above $\mathcal{F}_{\mathrm{D}} \sim 1 \mathrm{~mJ} / \mathrm{cm}^{2}$ the majority of the absorbed optical energy is on an $\sim 100$-fs timescale transferred to the strongly coupled optical phonons. This is corroborated with a quick initial recovery of the anisotropy (Fig. 6) that indicates that $T_{\mathrm{e}}$ drops below $\sim 300 \mathrm{~K}$ into the region of strong nematic fluctuations already a few hundred femtoseconds after the arrival of the D pulse. Although the increase in $\eta$ appears correlated with the observed optical nonlinearity we could not come up with any persuasive physical picture to explain the effect so we leave it open for further experimental confirmation and discussion.

\section{Destruction timescale}

The experimental destruction timescale of $\sim 150 \mathrm{fs}$ could be set either by the intrinsic low-energy SDW order parameter dynamics or, in the case of the fast adiabatic SDW order parameter, the finite initial NEDF thermalization timescale. Although the slower intrinsic SDW order dynamics on the $\sim 150$-fs timescale would not contradict the 3TM simulation results, the dependence of the destruction timescale on the Dphoton energy in $\mathrm{Sr}-122$ suggests that the destruction timescale is set by the initial NEDF thermalization.

A similar ultrafast SDW order destruction has been recently reported in chromium thin films where time-resolved ARPES [10] indicates that the SDW order parameter adiabatically follows the NEDF that appears completely thermalized within the 130 -fs experimental time-resolution window.

\section{E. Determination of the SDW-destruction threshold}

As in superconductors and charge-density waves [59] we associate the saturation of the transient reflectivity amplitude 
in the standard pump-probe experiments with the destruction of the ordered state. In the present case the saturation is incomplete where the finite slope at high-excitation density presumably corresponds to the transient response of the normal unordered state.

The shape of the saturation curve [see Figs. 2(c) and 2(g)] depends on the SDW-destruction threshold excitation energy density $U_{\text {th }}$, the geometrical parameters of the pump and probe beams, and their penetration depths [60]. In addition, the contribution of the pump-absorption saturation has to be taken into account in the present case.

To take into account the above effects we formulate a simple phenomenological saturation model where we approximate the local amplitude of the transient change in the dielectric constant $\Delta \epsilon(r, z)$ by a piecewise linear function of the locally absorbed energy density $U(r, z)$ that has different slopes below and above $U_{\text {th }}$,

$$
\begin{aligned}
\Delta \epsilon(r, z) & =\Delta \epsilon_{0} g(r, z), \\
g(r, z) & = \begin{cases}\frac{U(r, z)}{U_{\mathrm{th}}}, & U(r, z)<U_{\mathrm{th}}, \\
1+a\left(\frac{U(r, z)}{U_{\mathrm{th}}}-1\right), & U(r, z) \geqslant U_{\mathrm{th}},\end{cases}
\end{aligned}
$$

where $r$ corresponds to the radial distance from the beam center, $z$ is the normal distance from the sample surface, and $a$ is the relative slope in the normal state. The spatial dependence of $U(r, z)$ is given by

$$
\begin{gathered}
\frac{U(r, z)}{U_{\mathrm{th}}}=\frac{\mathcal{F}_{0}}{\mathcal{F}_{\mathrm{th}}} \frac{\left(1+e^{-\alpha_{\mathrm{P}} L_{\mathrm{P}}\left(\mathcal{F}_{\mathrm{th}}, 0\right)}\right) e^{-2 r^{2} / \rho_{\mathrm{P}}^{2}}}{\left(1+e^{\alpha_{\mathrm{P}}\left[z-L_{\mathrm{P}}\left(\mathcal{F}_{0}, r\right)\right]}\right)} \\
L_{\mathrm{P}}(\mathcal{F}, r)=c_{\alpha} \mathcal{F} e^{-2 r^{2} / \rho_{\mathrm{P}}^{2}}
\end{gathered}
$$

where $\alpha_{\mathrm{P}}$ is the linear pump-absorption coefficient. We phenomenologically take into account the pump-absorption saturation by using the Fermi function to model the $U(r, z)$ depth dependence introducing the local fluence-dependent pump-penetration depth (6). The coefficient $c_{\alpha}$ is determined from the multipulse experiment fits discussed above, whereas the pump beam is characterized by the pump beam diameter $\rho_{\mathrm{P}}$ and the external fluence in the center of the beam $\mathcal{F}_{0} . \mathcal{F}_{\text {th }}$ corresponds to the external threshold fluence at which $U_{\text {th }}$ is reached at the surface $(z=0)$ in the center of the beam $(r=0)$.

In the case of a relatively wide [61] Gaussian probe beam with diameter $\rho_{\operatorname{Pr}}$ Eq. (3) describing the transient-reflectivity amplitude can be simply upgraded to take into account the radial variation of the response (see also the Appendix),

$$
\begin{aligned}
A \propto & \int_{0}^{\infty} r d r \int_{0}^{\infty} d z e^{-2 r^{2} / \rho_{\mathrm{Pr}}^{2}} e^{-\alpha_{\mathrm{Pr}} z} \\
& \times \cos \left(2 n \frac{\omega_{0}}{c_{0}} z-\phi\right) g(r, z) .
\end{aligned}
$$

When fitting Eq. (7) to the experimental data it turns out that $\mathcal{F}_{\text {th }}, \phi$, and $a$ are strongly correlated. Since $\phi$ is usually not known a priori we fix $\phi$ to either 0 or $\pi / 2$ to obtain a range of values for $\mathcal{F}_{\text {th }}$. Example fits with $\phi=\pi / 2$, are shown [62] in Figs. 2(c) and 2(g) with the resulting $\mathcal{F}_{\text {th }}$ shown in Table II. Although the variation of $\phi$ can strongly influence the extracted $\mathcal{F}_{\text {th }}$, the determined ranges of $\mathcal{F}_{\text {th }}$ are very similar in both samples at both pump-photon energies.
TABLE II. The external destruction threshold fluence $\mathcal{F}_{\text {th }}$ at different pump-photon energies $\hbar \omega_{\mathrm{P}}$ for two extreme phase shifts $\phi$. The static optical constants used in fits were taken from Refs. [34,35].

\begin{tabular}{lcccc}
\hline \hline$\hbar \omega_{\mathrm{P}}$ & \multicolumn{3}{c}{$1.55 \mathrm{eV}$} & \multicolumn{2}{c}{$3.1 \mathrm{eV}$} \\
\hline$\phi$ & 0 & $\pi / 2$ & 0 & $\pi / 2$ \\
& \multicolumn{4}{c}{$\mathcal{F}_{\text {th }}\left(\mathrm{mJ} / \mathrm{cm}^{2}\right)$} \\
$\mathrm{EuFe}_{2} \mathrm{As}_{2}$ & 0.21 & 0.15 & 0.18 & 0.12 \\
$\mathrm{SrFe}_{2} \mathrm{As}_{2}$ & 0.21 & 0.16 & 0.20 & 0.16 \\
\hline \hline
\end{tabular}

Taking $\phi=\pi / 2$ indicated by the 3TM simulations (Table II) we calculate the destruction threshold energy density $U_{\text {th }} \sim 1.6 \mathrm{~kJ} / \mathrm{mol}$ for Eu-122 and $U_{\text {th }} \sim 2.5 \mathrm{~kJ} / \mathrm{mol}$ for Sr122. Assuming that $U_{\text {th }}$ corresponds to the condensation energy and taking $\gamma_{\mathrm{e}}$ from the 3TM fits we can estimate the SDW gap using the standard BCS formula and obtain $2 \Delta_{\mathrm{SDW}} / k_{\mathrm{B}} T_{\mathrm{SDW}}=$ 5 and 6 for Eu-122 and Sr-122, respectively. This is somewhat lower than the earlier weak-excitation pump-probe estimate [24] of 13 and 8 for Eu-122 and Sr-122, respectively, but closer to the optical conductivity result of 5.6 in Eu-122 [34].

Contrary to the superconductors $[59,63]$ there is no indication that the optical destruction energy would significantly exceed the estimated SDW condensation energy. This is consistent with the $3 \mathrm{TM}$ trajectories fit results where, at small $\mathcal{F}_{\mathrm{D}}$, that is comparable to $\mathcal{F}_{\text {th }}$, the fast optical energy transfer to the phonons is rather small (Fig. 10).

\section{SUMMARY AND CONCLUSIONS}

We presented an extensive all-optical study of the transient SDW state suppression and recovery in $\mathrm{EuFe}_{2} \mathrm{As}_{2}$ and $\mathrm{SrFe}_{2} \mathrm{As}_{2}$ under strong ultrafast optical excitation by means of the standard time-resolved pump probe as well as the multipulse transient optical spectroscopy.

The SDW order is suppressed on an $\sim 150$ - to $\sim 250$-fs timescale after a 50-fs destruction optical pulse absorption, depending on the optical-photon energy. The suppression timescale is fluence independent and set by the initial electronic thermalization timescale.

The SDW recovery timescale increases with the destruction optical-pulse fluence but remains below $\sim 1$ ps up to the fluence at which the transient lattice temperature exceeds the SDW transition temperature.

The optical SDW-destruction threshold energy densities of $\sim 1.6$ and $\sim 2.5 \mathrm{~kJ} / \mathrm{mol}$ in $\mathrm{EuFe}_{2} \mathrm{As}_{2}$ and $\mathrm{SrFe}_{2} \mathrm{As}_{2}$, respectively, are consistent with the BCS condensation energy estimates.

The time evolution of the multipulse system trajectories in a broad destruction-pulse fluence range can be well described within the framework of an extended three-temperature model assuming a fast sub-200-fs intrinsic order parameter timescale similar as recently observed for SDW in chromium [10]. The model fits indicate the normal-state specific-heat constant $\gamma_{\mathrm{e}}$ in the (50-60)- $\mathrm{mJ}^{-1} \mathrm{~mol}^{-1} \mathrm{~K}^{2}$ range. The fluencedependent recovery timescale is found to be governed by the optical-phonons-lattice-relaxation bottleneck that is strongly suppressed at high-excitation densities. The suppression of 
the bottleneck is attributed to a suppression of the nematicfluctuations-induced pseudogap at high temperatures.

The observed resilience of the SDW state at high fluences exceeding the SDW-destruction threshold fluence of $\sim 0.15 \mathrm{~mJ} / \mathrm{cm}^{2}$ up to $\sim 10$ times is attributed to saturation of the optical absorption. The model fits also suggest that at these fluences the majority of the absorbed optical energy is transferred to the optical phonons during the initial electronic thermalization on a few-hundred-femtosecond timescale.

\section{ACKNOWLEDGMENTS}

The authors acknowledge financial support from the Slovenian Research Agency (Research Core Funding No. No-P10040) and the European Research Council Advanced Grant TRAJECTORY (Grant No. GA 320602) for financial support.

\section{APPENDIX}

\section{Transient reflectivity}

Assuming that the beam diameters are large in comparison to the optical penetration depth and the transient dielectric constant varies slowly on the optical pulse timescale $\Delta \epsilon(z, t) \sim$ $\Delta \epsilon(z)$, we can write the wave equation for the perturbed probe field in one dimension,

$$
\frac{\mathcal{N}^{2}}{c_{0}^{2}} \frac{\partial^{2} \Delta E}{\partial t^{2}}-\frac{\partial^{2} \Delta E}{\partial z^{2}}=-\frac{1}{\epsilon_{0} c_{0}^{2}} \frac{\partial^{2} \Delta P}{\partial t^{2}},
$$

where $t$ is time, $z$ is the distance from the sample surface, $\mathcal{N}=n+i \kappa$ is the complex refraction index, $\Delta P$ is the pump-probe-induced transient polarization with $c_{0}^{2}$ and $\epsilon_{0}$ as the speed of light and the vacuum permittivity, respectively. In the presence of a monochromatic probe field, $E_{\operatorname{Pr}}(z, t)=$ $t_{12} E_{0 \mathrm{Pr}} e^{-i(\omega t-\mathcal{N} k z)}+$ c.c., propagating into the sample the transient polarization is given by

$$
\begin{aligned}
\Delta P(z, t) & =\epsilon_{0} \Delta \epsilon(z) E_{\operatorname{Pr}}(z, t), \\
& =t_{12} E_{0 \mathrm{Pr}} \epsilon_{0} \Delta \epsilon(z) \exp \left[-i\left(\omega t-\mathcal{N} \frac{\omega}{c_{0}} z\right)\right]+\text { c.c. }
\end{aligned}
$$

where $E_{0 \mathrm{Pr}}$ is the complex amplitude of the incident probe field at the sample surface (at $z=0)$ and $t_{12}=2 /(1+\mathcal{N}$ ) is the Fresnel transmission coefficient. Solving (A1) assuming (A2) we obtain, to the linear order in $\Delta \epsilon(z)$, the transient reflected field outside of the sample (at $z=0$ ),

$$
\Delta E_{\mathrm{r}}=\frac{i}{2} \frac{t_{12} t_{21} \omega}{c_{0} \mathcal{N}} E_{\operatorname{Pr} 0} e^{-i \omega t} \int_{0}^{\infty} \Delta \epsilon(z) e^{2 i \mathcal{N}\left(\omega / c_{0}\right) u} d z+\text { c.c. }
$$

where the Fresnel coefficient $t_{21}=2 \mathcal{N} /(1+\mathcal{N})$ takes into account the transmission from the sample to vacuum.

In the case of an incident Gaussian probe pulse,

$$
\begin{aligned}
E_{\mathrm{Pr}}^{\mathrm{pulse}}(t) & =A_{0} \sqrt{\frac{2}{\tau \sqrt{\pi}}} e^{-2 t^{2} / \tau^{2}} e^{-i \omega_{0} t}+\text { c.c. } \\
& =\int_{0}^{\infty} A\left(\omega-\omega_{0}\right) e^{-i \omega t} d \omega+\text { c.c. },
\end{aligned}
$$

the total transient reflected electric field, neglecting the dispersion, is the integral,

$$
\begin{aligned}
\Delta E_{\mathrm{r}}^{\mathrm{pulse}}(t)= & \frac{i}{2} \frac{t_{12} t_{21}}{c_{0} \mathcal{N}} \int_{0}^{\infty} d z \Delta \epsilon(z) \int_{0}^{\infty} d \omega \omega A\left(\omega-\omega_{0}\right) e^{-i \omega t} e^{2 i \mathcal{N}\left(\omega / c_{0}\right) z}+\text { c.c. } \\
= & -\frac{t_{12} t_{21} A_{0}}{2 \sqrt{2} \sqrt[4]{\pi} c_{0} \mathcal{N} \sqrt{\tau}} \frac{\partial}{\partial t^{\prime}}\left[\int _ { 0 } ^ { \infty } \Delta \epsilon ( z ) e ^ { - 2 t ^ { \prime 2 } / \tau ^ { 2 } } \left\{e^{-i \omega_{0} t^{\prime}}\left(1+\operatorname{erf}\left[\frac{\omega_{0} \tau}{2 \sqrt{2}}+i \sqrt{2} \frac{t^{\prime}}{\tau}\right]\right)\right.\right. \\
& \left.\left.+e^{i \omega_{0} t^{\prime}}\left(1-\operatorname{erf}\left[\frac{\omega_{0} \tau}{2 \sqrt{2}}-i \sqrt{2} \frac{t^{\prime}}{\tau}\right]\right)\right\} d z\right]+ \text { c.c. } \\
\approx & -\frac{t_{12} t_{21} A_{0}}{2 \sqrt[4]{\pi} c_{0} \mathcal{N} \sqrt{\tau}} \frac{\partial}{\partial t}\left[\int_{0}^{\infty} \Delta \epsilon(z) e^{-2\left(t-2 \mathcal{N} z / c_{0}\right)^{2} / \tau^{2}} e^{-i \omega_{0}\left(t-2 \mathcal{N} z / c_{0}\right)} d u\right]+\text { c.c. },
\end{aligned}
$$

where $t^{\prime}=t-2 \mathcal{N} z / c_{0}$. In the last line we assumed that the pulse is narrow-band $\omega_{0} \tau \gg 1$ so

$$
\left|t^{\prime}\right| / \tau \ll \omega_{0} \tau .
$$

The transient reflectivity is then given by

$$
\begin{aligned}
\frac{\Delta R}{R}=\frac{\Delta I_{\mathrm{r}}}{I_{\mathrm{r}}} \simeq \frac{2 \int \operatorname{Re}\left(\Delta E_{\mathrm{r}}^{\mathrm{pulse}} E_{\mathrm{r}}^{*}\right) d t}{\int E_{\mathrm{r}} E_{\mathrm{r}}^{*} d t} \\
=\operatorname{Re}\left[-\frac{t_{12} t_{21}}{\sqrt{\pi} c_{0} r_{12} \mathcal{N} \tau} \int d t e^{-2 t^{2} / \tau^{2}} e^{i \omega_{0} t} \times \frac{\partial}{\partial t} \int_{0}^{\infty} d z \Delta \epsilon(z) e^{i \omega_{0}\left(2 \mathcal{N} z / c_{0}-t\right)} e^{-2\left(2 \mathcal{N} z / c_{0}-t\right)^{2} / \tau^{2}}\right] \\
=\operatorname{Re}\left[-\frac{\omega_{0} t_{12} t_{21}}{2 c_{0} r_{12} \mathcal{N}} \int_{0}^{\infty} d z \Delta \epsilon(z) e^{2 i\left(\omega_{0} / c_{0}\right) \mathcal{N} z} K(z)\right], \\
K(z)=\left(1+i \frac{4 \mathcal{N} z}{\omega_{0} c_{0} \tau^{2}}\right) e^{-4 \mathcal{N}^{2} z^{2} / c_{0}^{2} \tau^{2}},
\end{aligned}
$$


where $E_{\mathrm{r}}=r_{12} E_{\mathrm{Pr}}^{\text {pulse }}(t)$ and $r_{12}=(1-\mathcal{N}) /(1+\mathcal{N})$ is the reflection coefficient. In comparison to the continuous wave case (A3) an additional term $K(z)$ appears in the kernel of the integral (A7). Due to the exponent the kernel in (A7) decays on the length scale $z \sim c_{0} / \omega_{0} \kappa$ satisfying the condition (A6) when $\omega_{0} \tau \gg \sqrt{n^{2} / \kappa^{2}+1}$. On this length scale the argument of the exponent in (A8) is on the order of $4|\mathcal{N}|^{2} / \kappa^{2} \omega_{0}^{2} \tau^{2} \ll 1$ for the narrow-band pulses satisfying $\omega_{0} \tau \gg \sqrt{n^{2} / \kappa^{2}+1}$ so $K(z) \sim 1$ can be dropped from (A7).

Due to the phase factor in (A7) the real and imaginary parts of $\Delta \epsilon(z)=\Delta \epsilon_{\mathrm{r}}(z)+i \Delta \epsilon_{\mathrm{i}}(z)$ show different depth sensitivities that depend on the static complex refraction index,

$$
\begin{aligned}
\frac{\Delta R}{R} & =\frac{4 \omega_{0}}{c_{0}\left|\mathcal{N}^{2}-1\right|} \int_{0}^{\infty} d z e^{-\alpha_{\mathrm{Pr}} z}\left[\Delta \epsilon_{\mathrm{r}}(z) \sin \left(2 n \frac{\omega_{0}}{c_{0}} z-\beta\right)+\Delta \epsilon_{\mathrm{i}}(z) \cos \left(2 n \frac{\omega_{0}}{c_{0}} z-\beta\right)\right], \\
\tan (\beta) & =\frac{\operatorname{Im}\left(\mathcal{N}^{2}-1\right)}{\operatorname{Re}\left(\mathcal{N}^{2}-1\right)}=\frac{2 n \kappa}{n^{2}-\kappa^{2}-1},
\end{aligned}
$$

where $\alpha_{\mathrm{Pr}}=2 \kappa \frac{\omega_{0}}{c_{0}}$ is the probe-absorption coefficient.

Since in the simple saturation model (5) the real and imaginary parts of $\Delta \epsilon(z)$ are assumed to have the same $z$ dependence Eq. (A9) is simplified to

$$
\begin{gathered}
\frac{\Delta R}{R}=\frac{4 \omega_{0}\left|\Delta \epsilon_{0}\right|}{c_{0}\left|\mathcal{N}^{2}-1\right|} \int_{0}^{\infty} d z e^{-\alpha_{\mathrm{Pr}} z} \cos \left(2 n \frac{\omega_{0}}{c_{0}} z-\phi\right) g(z), \\
\tan (\phi)=\frac{2 n \kappa \Delta \epsilon_{0 \mathrm{r}}-\left(n^{2}-\kappa^{2}-1\right) \Delta \epsilon_{0 \mathrm{i}}}{2 n \kappa \Delta \epsilon_{0 \mathrm{i}}+\left(n^{2}-\kappa^{2}-1\right) \Delta \epsilon_{0 \mathrm{r}}}
\end{gathered}
$$

In the case of Gaussian pump and probe beams with the diameters $\rho_{\mathrm{P}}$ and $\rho_{\mathrm{Pr}}$, respectively, (A10) can be easily extended [64] to (7) by an additional integration in the radial direction [65] where $g(r, z)$ is obtained from (5) by taking into account the radial pump fluence dependence.

With increasing pump fluence the boundary between the ordered- and the normal-state regions in (5) moves along $z$, so the oscillatory factor in the integral can lead to a nonmonotonous excitation-density dependence of the $\Delta R / R$ when $n / \kappa \gtrsim 1$ as shown in Fig. 11. There is unfortunately no clear singularity observed when the threshold fluence is reached at low $-(n / \kappa)$ ratios. Moreover, the saturation is much less pronounced for $\phi=\pi / 2$.
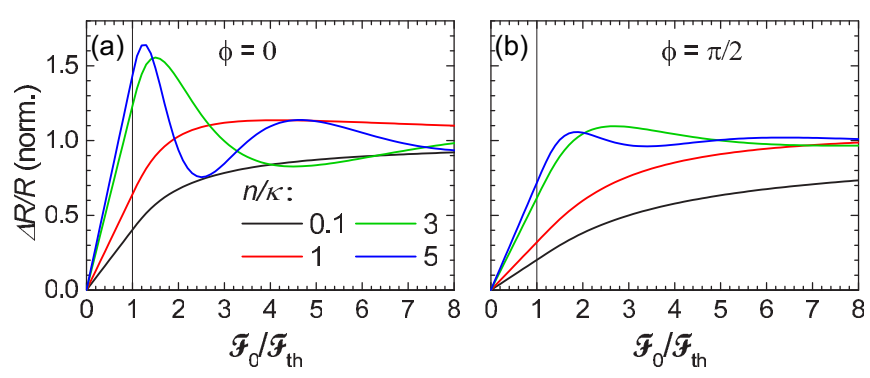

FIG. 11. Simulated fluence dependence for the case of Gaussian beams from Eq. (7) for different ratios $n / \kappa$ for two orthogonal phase shifts $\phi$ taking $\rho_{\mathrm{P}} / \rho_{\mathrm{Pr}}=2, \alpha_{\mathrm{Pr}} / \alpha_{\mathrm{P}}=1, c_{\alpha}=0$, and $a=0$. The curves are normalized to the saturated value at $\mathcal{F}_{0} \gg \mathcal{F}_{\text {th }}$.

\section{Three-temperature model}

The time evolution of the temperatures in the threetemperature model is governed by

$$
\begin{aligned}
\gamma_{\mathrm{e}} T_{\mathrm{e}} \frac{\partial T_{\mathrm{e}}}{\partial t} & =(1-\eta) s(z, t)-G_{\mathrm{eo}}\left(T_{\mathrm{e}}-T_{\mathrm{o}}\right)+\kappa \frac{\partial T_{\mathrm{e}}^{2}}{\partial z^{2}}, \\
c_{\mathrm{E}}\left(T_{\mathrm{o}}\right) \frac{\partial T_{\mathrm{o}}}{\partial t} & =\eta s(z, t)+G_{\mathrm{eo}}\left(T_{\mathrm{e}}-T_{\mathrm{o}}\right)-G_{\mathrm{ol}}\left(T_{\mathrm{o}}-T_{1}\right), \\
c_{\mathrm{L}}\left(T_{\mathrm{L}}\right) \frac{\partial T_{\mathrm{L}}}{\partial t} & =G_{\mathrm{ol}}\left(T_{\mathrm{o}}-T_{\mathrm{L}}\right),
\end{aligned}
$$

where $T_{\mathrm{e}}, T_{\mathrm{o}}$, and $T_{\mathrm{L}}$ are the electronic, the strongly coupled $\mathrm{OP}$, and lattice temperatures, respectively (see Fig. 12). $\gamma_{\mathrm{e}}$ is the normal-state electronic specific-heat constant [66], $c_{\mathrm{E}}\left(T_{\mathrm{o}}\right)$ is the Einstein phonon specific heat, and $c_{\mathrm{L}}\left(T_{\mathrm{L}}\right)$ is the lattice

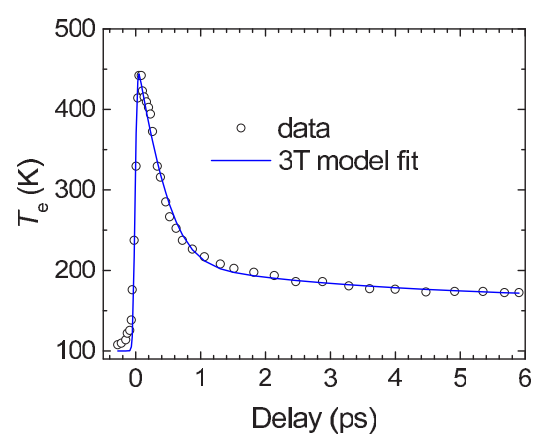

FIG. 12. Three-temperature model fit to the electronic temperature in Eu-122 from TR-ARPES [46]. Setting $\eta$ to different values results in virtually identical fit curves (not shown) with modified remaining 3TM parameters (see Table I). 

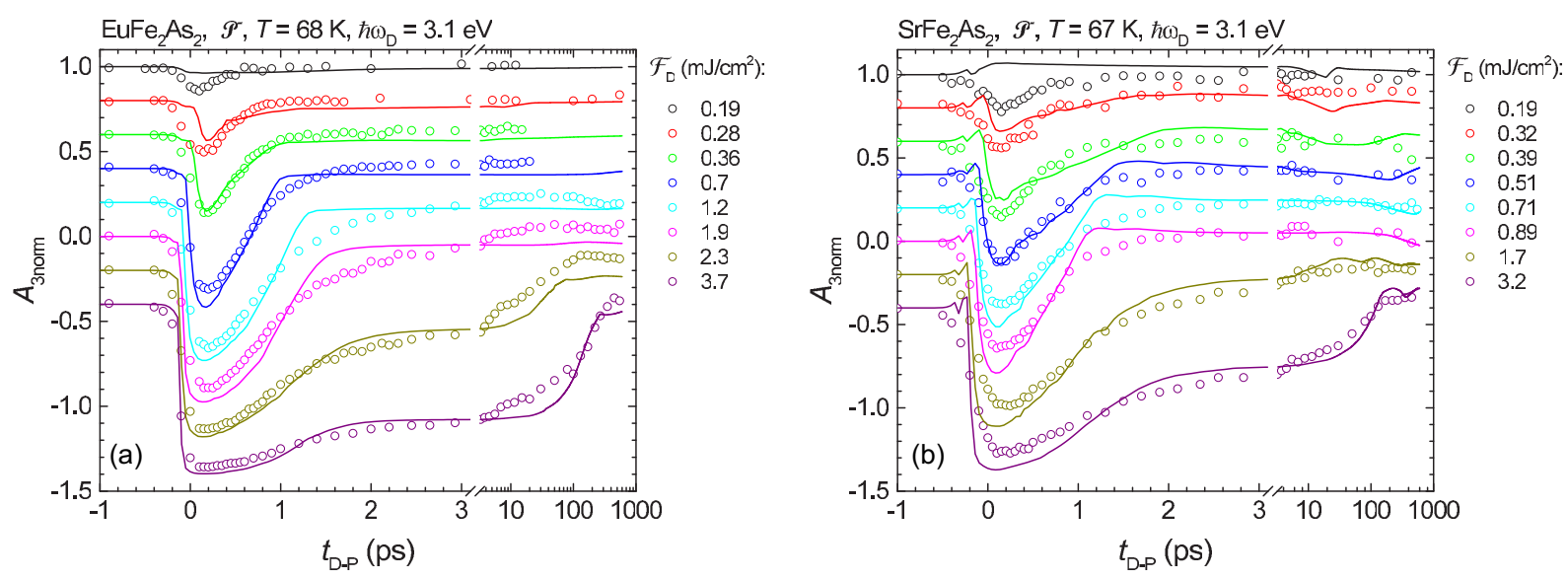

FIG. 13. Comparison of the experimental and simulated trajectories for the cases of $\eta=0.01$ and $\phi=\pi / 2$. The trajectories at different $\mathcal{F}_{\mathrm{D}}$ 's are vertically shifted for clarity.

specific heat. $G_{\mathrm{eo}}$ and $G_{\mathrm{ol}}$ are the electron-OP and OP-lattice coupling constants, whereas $\kappa$ is the electronic heat diffusivity. $s(z, t)$ is the absorbed laser energy density rate. Using the branching factor $\eta$, we take into account that the primary electron-hole pair can relax by exciting the optical phonons during the thermalization.

To take into account absorption saturation we approximate $s(z, t)$ by

$$
s(z, t) \propto \mathcal{F}_{\mathrm{D}} e^{-\left(2 t^{2} / \tau_{\mathrm{p}}^{2}\right)}\left[1+e^{\alpha_{\mathrm{D}}\left(z-L_{\mathrm{D}}\right)}\right]^{-1},
$$

where $\tau_{\mathrm{p}}$ is the effective heating pulse length, $\alpha_{\mathrm{D}}$ is the D-pulse linear absorption coefficient, and $L_{\mathrm{D}}$ is the phenomenological absorption length.

$c_{\mathrm{E}}(T)$ is parametrized by the Einstein model,

$$
c_{\mathrm{E}}(T)=c_{\mathrm{E} 0}\left(\frac{T_{\mathrm{E}}}{T}\right)^{2} e^{T_{\mathrm{E}} / T} /\left(e^{T_{\mathrm{E}} / T}-1\right)^{2}
$$

whereas $c_{\mathrm{L}}(T)$ is obtained from the total experimental specificheat-capacity $[32,33] c_{\mathrm{p}}(T)$ by subtracting the electronic and
OP parts,

$$
c_{\mathrm{L}}(T)=c_{\mathrm{p}}(T)-\gamma_{\mathrm{e}} T_{\mathrm{e}}-c_{\mathrm{E}}(T) .
$$

According to Allen [54] the second moment of the Eliashberg function can be expressed as

$$
\lambda\left\langle\omega^{2}\right\rangle=\frac{\pi k_{\mathrm{B}}}{3 \hbar} \frac{G_{\mathrm{eo}}}{\gamma_{\mathrm{e}}} .
$$

\section{Three-temperature model fits with a single $\mathcal{F}_{\text {D-dependent parameter }}$}

A worse fit, particularly at low $\mathcal{F}_{\mathrm{D}}$, with fixed and $\mathcal{F}_{\mathrm{D}}-$ independent $\eta$ shown in Fig. 13, results in the parameters shown in Table III. The increase in $G_{\mathrm{ol}}$ with $\mathcal{F}_{\mathrm{D}}$ appears to be robust with respect to how $\eta$ is fit (Fig. 14).

In Fig. 15 we plot example 3TM temperatures evolution obtained from the $\mathcal{F}_{\mathrm{D}}$-dependent $\eta$ fits in Fig. 9 and compare them with the fixed $\eta=0.01$ fits. There is a substantial difference in the $T_{\mathrm{e}}$ magnitude at short times. This is a consequence of the response function saturation resulting from the fact that $A(T)$ is virtually zero above $T \sim 300 \mathrm{~K}$. In the large $\eta$ case

\begin{tabular}{|c|c|c|c|c|c|c|c|c|}
\hline & $\eta^{\mathrm{a}}$ & 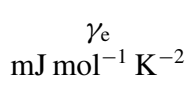 & 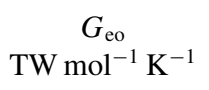 & $\begin{array}{c}\lambda\left\langle\omega^{2}\right\rangle \\
(\mathrm{meV})^{2}\end{array}$ & $\begin{array}{c}c_{\mathrm{E} 0}^{\mathrm{b}} \\
\mathrm{J} \mathrm{mol}^{-1} \mathrm{~K}^{-1}\end{array}$ & $\begin{array}{c}G_{\mathrm{ol}} \\
\mathrm{TW} \mathrm{\textrm {mol } ^ { - 1 }} \mathrm{K}^{-1}\end{array}$ & $\begin{array}{l}k=\kappa / V_{\mathrm{mol}} \\
\mathrm{W} \mathrm{m}^{-1} \mathrm{~K}^{-1}\end{array}$ & $\begin{array}{c}L_{\mathrm{D}} / \mathcal{F}_{\mathrm{D}}^{\mathrm{c}} \\
\mathrm{nm} \mathrm{cm} \mathrm{mJ}^{-1}\end{array}$ \\
\hline \multirow{2}{*}{$\begin{array}{l}\text { Eu-122 (TR-ARPES }) \\
{[46](T \sim 100 \mathrm{~K})^{\mathrm{d}}}\end{array}$} & 0.01 & $52 \pm 3$ & $34 \pm 3$ & $39 \pm 3$ & $67 \pm 14$ & $7 \pm 5$ & & 17 \\
\hline & 0.5 & $30 \pm 2$ & $23 \pm 3$ & $45 \pm 3$ & $76 \pm 20$ & $11 \pm 9$ & & 17 \\
\hline \multirow{2}{*}{$\begin{array}{l}\text { Eu-122 (present } \\
\text { paper })(T \sim 70 \mathrm{~K})\end{array}$} & 0.01 & $42 \pm 2$ & $96 \pm 6$ & $135 \pm 7$ & $15 \pm 1$ & Fig. 14 & $14 \pm 1$ & $17 \pm 5$ \\
\hline & 0.5 & $10 \pm 1$ & $138 \pm 6$ & $820 \pm 130$ & $21 \pm 1$ & & & \\
\hline \multirow{2}{*}{$\begin{array}{l}\text { Sr-122 (present } \\
\text { paper })(T \sim 70 \mathrm{~K})\end{array}$} & 0.01 & $68 \pm 4$ & $112 \pm 6$ & $97 \pm 6$ & $17 \pm 1$ & Fig. 14 & $10 \pm 1$ & $23 \pm 5$ \\
\hline & 0.5 & $38 \pm 1$ & $218 \pm 16$ & $340 \pm 20$ & $25 \pm 1$ & & & \\
\hline
\end{tabular}
$T_{\mathrm{o}}$ significantly exceeds $T_{\mathrm{e}}$ at short times similar as previously observed [50] in $\mathrm{MgB}_{2}$.

TABLE III. Comparison of the three-temperature model parameters obtained from fits with $\mathcal{F}_{\mathrm{D}}$-dependent $G_{\mathrm{ol}}$.

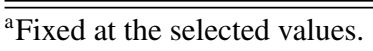

${ }^{\mathrm{b}} T_{\mathrm{E}}$ was without fitting set to $300 \mathrm{~K}$.

${ }^{\mathrm{c}}$ Obtained from the two highest fluences fit in the multipulse case.

${ }^{\mathrm{d}} \mathcal{F}_{\mathrm{D}} \sim 1 \mathrm{~mJ} / \mathrm{cm}^{2}$. 


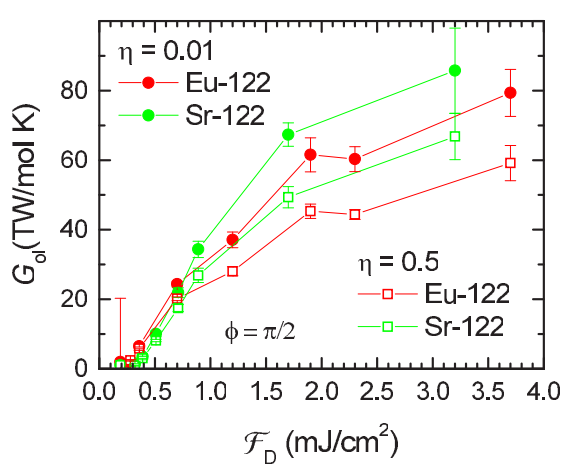

FIG. 14. The destruction-pulse fluence dependence of the electron-optical-phonons coupling from the 3TM fit for two different fixed branching factors.
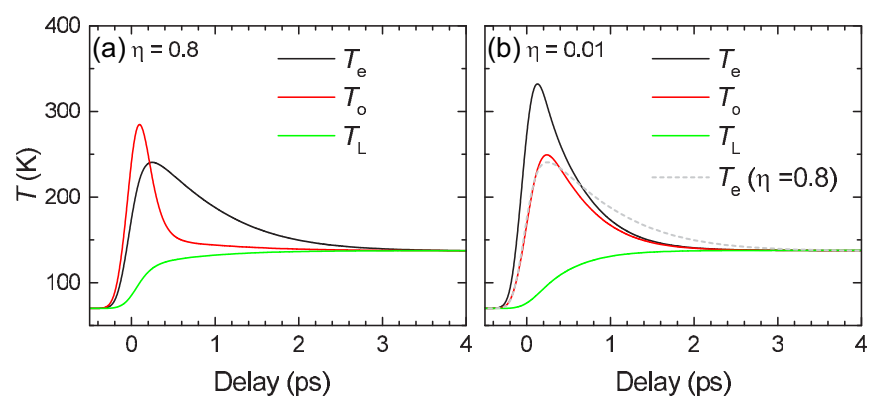

FIG. 15. The time evolution of the 3TM temperatures at $\alpha_{\mathrm{Pr}} z=1$ from fits for Eu-122 at $\mathcal{F}_{\mathrm{D}}=1.2 \mathrm{~mJ} / \mathrm{cm}^{2}$. Panel (a) corresponds to the $\mathcal{F}_{\mathrm{D}}=1.2 \mathrm{~mJ} / \mathrm{cm}^{2}$ fit in Fig. 9, and panel (b) corresponds to the corresponding fit in Fig. 13.
[1] S. Iwai, K. Yamamoto, A. Kashiwazaki, F. Hiramatsu, H. Nakaya, Y. Kawakami, K. Yakushi, H. Okamoto, H. Mori, and Y. Nishio, Phys. Rev. Lett. 98, 097402 (2007).

[2] C. Kübler, H. Ehrke, R. Huber, R. Lopez, A. Halabica, R. F. Haglund, Jr., and A. Leitenstorfer, Phys. Rev. Lett. 99, 116401 (2007).

[3] F. Schmitt, P. S. Kirchmann, U. Bovensiepen, R. G. Moore, L. Rettig, M. Krenz, J.-H. Chu, N. Ru, L. Perfetti, D. Lu et al., Science 321, 1649 (2008).

[4] A. Tomeljak, H. Schäfer, D. Städter, M. Beyer, K. Biljakovic, and J. Demsar, Phys. Rev. Lett. 102, 066404 (2009).

[5] J. C. Petersen, S. Kaiser, N. Dean, A. Simoncig, H. Liu, A. L. Cavalieri, C. Cacho, I. Turcu, E. Springate, F. Frassetto et al., Phys. Rev. Lett. 107, 177402 (2011).

[6] F. Schmitt, P. S. Kirchmann, U. Bovensiepen, R. Moore, J. Chu, D. Lu, L. Rettig, M. Wolf, I. Fisher, and Z. Shen, New J. Phys. 13, 063022 (2011).

[7] P. Beaud, A. Caviezel, S. Mariager, L. Rettig, G. Ingold, C. Dornes, S. Huang, J. Johnson, M. Radovic, T. Huber et al., Nature Mater. 13, 923 (2014).

[8] C. L. Smallwood, W. Zhang, T. L. Miller, C. Jozwiak, H. Eisaki, D.-H. Lee, and A. Lanzara, Phys. Rev. B 89, 115126 (2014).

[9] I. Madan, P. Kusar, V. V. Baranov, M. Lu-Dac, V. V. Kabanov, T. Mertelj, and D. Mihailovic, Phys. Rev. B 93, 224520 (2016).

[10] C. W. Nicholson, C. Monney, R. Carley, B. Frietsch, J. Bowlan, M. Weinelt, and M. Wolf, Phys. Rev. Lett. 117, 136801 (2016).

[11] H. Ichikawa, S. Nozawa, T. Sato, A. Tomita, K. Ichiyanagi, M. Chollet, L. Guerin, N. Dean, A. Cavalleri, S.-i. Adachi et al., Nature Mater. 10, 101 (2011).

[12] L. Stojchevska, I. Vaskivskyi, T. Mertelj, P. Kusar, D. Svetin, S. Brazovskii, and D. Mihailovic, Science 344, 177 (2014).

[13] V. R. Morrison, R. P. Chatelain, K. L. Tiwari, A. Hendaoui, A. Bruhács, M. Chaker, and B. J. Siwick, Science 346, 445 (2014).

[14] Y. Kamihara, H. Hiramatsu, M. Hirano, R. Kawamura, H. Yanagi, T. Kamiya, and H. Hosono, J. Am. Chem. Soc. 128, 10012 (2006)

[15] Y. Kamihara, T. Watanabe, M. Hirano, H. Hosono et al., J. Am. Chem. Soc 130, 3296 (2008).

[16] G. R. Stewart, Rev. Mod. Phys. 83, 1589 (2011).
[17] T.-M. Chuang, M. P. Allan, J. Lee, Y. Xie, N. Ni, S. L. Bud'ko, G. S. Boebinger, P. C. Canfield, and J. C. Davis, Science 327, 181 (2010).

[18] J.-H. Chu, J. G. Analytis, K. De Greve, P. L. McMahon, Z. Islam, Y. Yamamoto, and I. R. Fisher, Science 329, 824 (2010).

[19] A. Dusza, A. Lucarelli, F. Pfuner, J.-H. Chu, I. R. Fisher, and L. Degiorgi, Europhys. Lett. 93, 37002 (2011).

[20] T. Mertelj, P. Kusar, V. V. Kabanov, L. Stojchevska, N. D. Zhigadlo, S. Katrych, Z. Bukowski, J. Karpinski, S. Weyeneth, and D. Mihailovic, Phys. Rev. B 81, 224504 (2010).

[21] L. Stojchevska, P. Kusar, T. Mertelj, V. V. Kabanov, X. Lin, G. H. Cao, Z. A. Xu, and D. Mihailovic, Phys. Rev. B 82, 012505 (2010).

[22] L. Rettig, R. Cortés, S. Thirupathaiah, P. Gegenwart, H. S. Jeevan, M. Wolf, J. Fink, and U. Bovensiepen, Phys. Rev. Lett. 108, 097002 (2012).

[23] K. W. Kim, A. Pashkin, H. Schäfer, M. Beyer, M. Porer, T. Wolf, C. Bernhard, J. Demsar, R. Huber, and A. Leitenstorfer, Nature Mater. 11, 497 (2012).

[24] A. Pogrebna, N. Vujičič, T. Mertelj, T. Borzda, G. Cao, Z. A. Xu, J.-H. Chu, I. R. Fisher, and D. Mihailovic, Phys. Rev. B 89, 165131 (2014).

[25] A. Patz, T. Li, S. Ran, R. M. Fernandes, J. Schmalian, S. L. Bud'ko, P. C. Canfield, I. E. Perakis, and J. Wang, Nat. Commun. 5, 3229 (2014).

[26] S. Gerber, K. Kim, Y. Zhang, D. Zhu, N. Plonka, M. Yi, G. Dakovski, D. Leuenberger, P. Kirchmann, R. Moore et al., Nat. Commun. 6, 7377 (2015).

[27] L. Rettig, S. O. Mariager, A. Ferrer, S. Grübel, J. A. Johnson, J. Rittmann, T. Wolf, S. L. Johnson, G. Ingold, P. Beaud, and U. Staub, Struct. Dyn. 3, 023611 (2016).

[28] R. Yusupov, T. Mertelj, V. V. Kabanov, S. Brazovskii, P. Kusar, J.-H. Chu, I. R. Fisher, and D. Mihailovic, Nat. Phys. 6, 681 (2010).

[29] M. Tegel, M. Rotter, V. Weiß, F. M. Schappacher, R. Pöttgen, and D. Johrendt, J. Phys.: Condens. Matter 20, 452201 (2008).

[30] A. Pogrebna, T. Mertelj, G. Cao, Z. A. Xu, and D. Mihailovic, Phys. Rev. B 94, 144519 (2016).

[31] The electronic system and the lattice are expected to be in the local thermal equilibrium on this timescale. 
[32] J. Herrero-Martín, V. Scagnoli, C. Mazzoli, Y. Su, R. Mittal, Y. Xiao, T. Brueckel, N. Kumar, S. K. Dhar, A. Thamizhavel, and L. Paolasini, Phys. Rev. B 80, 134411 (2009).

[33] G. F. Chen, Z. Li, J. Dong, G. Li, W. Z. Hu, X. D. Zhang, X. H. Song, P. Zheng, N. L. Wang, and J. L. Luo, Phys. Rev. B 78, 224512 (2008).

[34] D. Wu, N. Barišić, N. Drichko, S. Kaiser, A. Faridian, M. Dressel, S. Jiang, Z. Ren, L. J. Li, G. H. Cao, Z. A. Xu, H. S. Jeevan, and P. Gegenwart, Phys. Rev. B 79, 155103 (2009).

[35] A. Charnukha, D. Pröpper, T. I. Larkin, D. L. Sun, Z. W. Li, C. T. Lin, T. Wolf, B. Keimer, and A. V. Boris, Phys. Rev. B 88, 184511 (2013).

[36] We obtain the light penetration depth of $\alpha_{\mathrm{Pr}}^{-1}=27$ and $24 \mathrm{~nm}$ in Eu-122 and 24 and $17 \mathrm{~nm}$ in $\mathrm{Sr}-122$ at $\hbar \omega=1.55$ and $\hbar \omega=$ $3.1 \mathrm{eV}$, respectively.

[37] In the absence of the $D$ pulse.

[38] In the absence of thermal transport data we approximate $c$-axis thermal conductivity using the Wiedmann-Franz law taking the measured interplane resistivity and heat-capacity data [33] in $\mathrm{SrFe}_{2} \mathrm{As}_{2}$ to obtain thermal diffusivity of $K_{z} \sim 0.01 \mathrm{~nm}^{2} / \mathrm{ps}$.

[39] The in-plane mean carrier free path in Sr-122 at low $T$ is on the order of a few $100 \mathrm{~nm}$ with the Fermi velocity of $\sim 5 \times 10^{4} \mathrm{~m} / \mathrm{s}$ [40]. Whereas on the 1-ps timescale, when the electronic system is still highly excited, the in-plane ballistic transport on the 100$\mathrm{nm}$ length scale would be possible, and the out-of-plane transport on a similar length scale at elevated $T \sim 100 \mathrm{~K}$ is impossible due to the substantial resistivity anisotropy [33] on the order of $\sim 100$ in Sr-122.

[40] M. Sutherland, D. J. Hills, B. S. Tan, M. M. Altarawneh, N. Harrison, J. Gillett, E. C. T. O'Farrell, T. M. Benseman, I. Kokanovic, P. Syers, J. R. Cooper, and S. E. Sebastian, Phys. Rev. B 84, 180506 (2011).

[41] L. Stojchevska, T. Mertelj, J.-H. Chu, I. R. Fisher, and D. Mihailovic, Phys. Rev. B 86, 024519 (2012).

[42] M. Yi, D. Lu, J.-H. Chu, J. G. Analytis, A. P. Sorini, A. F. Kemper, B. Moritz, S.-K. Mo, R. G. Moore, M. Hashimoto, W.-S. Lee, Z. Hussain, T. P. Devereaux, I. R. Fisher, and Z.-X. Shen, Proc. Natl. Acad. Sci. USA 108, 6878 (2011).

[43] I. Madan, V. V. Baranov, Y. Toda, M. Oda, T. Kurosawa, V. V. Kabanov, T. Mertelj, and D. Mihailovic, Phys. Rev. B 96, 184522 (2017).

[44] In the case of the Rothwarf-Taylor bottleneck model the parameter is the nonequilibrium quasiparticle density.
[45] G. Psaltakis, Solid State Commun. 51, 535 (1984).

[46] L. Rettig, R. Cortés, H. S. Jeevan, P. Gegenwart, T. Wolf, J. Fink, and U. Bovensiepen, New J. Phys. 15, 083023 (2013).

[47] Within the probe penetration depth of $\sim 1 / \alpha_{\mathrm{Pr}} \sim 30 \mathrm{~nm}$.

[48] L. Perfetti, P. A. Loukakos, M. Lisowski, U. Bovensiepen, H. Eisaki, and M. Wolf, Phys. Rev. Lett. 99, 197001 (2007).

[49] See Appendix Sec. 2 for the detailed formulation.

[50] J. Demsar, R. D. Averitt, A. J. Taylor, V. V. Kabanov, W. N. Kang, H. J. Kim, E. M. Choi, and S. I. Lee, Phys. Rev. Lett. 91, 267002 (2003).

[51] See Appendix Eq. (A13) for the formal definition.

[52] Setting $\mathcal{F}_{\mathrm{D}} \sim 0$ in (A13) leads to approximately exponential fluence decay with the penetration depth equal to the equilibrium optical penetration depth.

[53] Fits with $\phi=0$ show much worse agreement at low $\mathcal{F}_{\mathrm{D}}$.

[54] P. B. Allen, Phys. Rev. Lett. 59, 1460 (1987).

[55] We take $\gamma_{\mathrm{e}}$ measured in Sr-122 since in it is not experimentally accessible in Eu-122 due to the $\mathrm{Eu}^{2+}$ spin ordering at low $T$.

[56] F. Hardy, P. Burger, T. Wolf, R. A. Fisher, P. Schweiss, P. Adelmann, R. Heid, R. Fromknecht, R. Eder, D. Ernst, H. v. Löhneysen, and C. Meingast, Europhys. Lett. 91, 47008 (2010).

[57] D. C. Johnston, Adv. Phys. 59, 803 (2010).

[58] Possible excitation density dependence of the response function (2) could cause the worst fits using $\mathcal{F}_{\mathrm{D}}$-independent $\eta$.

[59] L. Stojchevska, P. Kusar, T. Mertelj, V. V. Kabanov, Y. Toda, X. Yao, and D. Mihailovic, Phys. Rev. B 84, 180507 (2011).

[60] The ordered state destruction is spatially nonuniform due to the inhomogeneous excitation profile.

[61] With respect to the optical wavelength.

[62] Contrary to the multipulse trajectories simulations (see Fig. 9) where taking $\phi=0$ resulted in a worse fit to the data, the $\phi=0$ fit curves are virtually identical to the $\phi=\pi / 2$ curves in this case.

[63] M. Beyer, D. Städter, M. Beck, H. Schäfer, V. V. Kabanov, G. Logvenov, I. Bozovic, G. Koren, and J. Demsar, Phys. Rev. B 83, 214515 (2011).

[64] P. Kusar, V. V. Kabanov, J. Demsar, T. Mertelj, S. Sugai, and D. Mihailovic, Phys. Rev. Lett. 101, 227001 (2008).

[65] When both diameters are much larger than the corresponding wavelengths.

[66] Since the response function (2) used for calculating the trajectories is virtually constant below $T_{\mathrm{SDW}}$ we can neglect the drop in $\gamma_{\mathrm{e}}$ in the SDW state. 